\title{
Photo-z optimization for measurements of the BAO radial scale
}

\author{
Daniel Roig ${ }^{1}$, Licia Verde ${ }^{3,1}$, Jordi Miralda-Escudé $e^{3,2,1}$, \\ Raul Jimenez ${ }^{3,1}$, Carlos Peña-Garay ${ }^{4}$ \\ ${ }^{1}$ Institute of Space Sciences (CSIC-IEEC), Fac. Ciències, Campus \\ UAB, Bellaterra, Spain. \\ 2 Institut of Ciències del Cosmos, Universitat de Barcelona, \\ Barcelona, Spain. \\ ${ }^{3}$ ICREA, Barcelona, Spain \\ ${ }^{4}$ Instituto de Física Corpuscular (CSIC-UVEG), València, Spain
}

\begin{abstract}
.
Baryon Acoustic Oscillations (BAO) in the radial direction offer a method to directly measure the Universe expansion history, and to set limits to space curvature when combined to the angular BAO signal. In addition to spectroscopic surveys, radial $\mathrm{BAO}$ might be measured from accurate enough photometric redshifts obtained with narrow-band filters. We explore the requirements for a photometric survey using Luminous Red Galaxies (LRG) to competitively measure the radial BAO signal and discuss the possible systematic errors of this approach. If LRG were a highly homogeneous population, we show that the photo-z accuracy would not substantially improve by increasing the number of filters beyond $\sim 10$, except for a small fraction of the sources detected at high signal-to-noise, and broad-band filters would suffice to achieve the target $\sigma_{z}=0.003(1+z)$ for measuring radial BAO. Using the LRG spectra obtained from SDSS, we find that the spectral variability of LRG substantially worsens the achievable photometric redshift errors, and that the optimal system consists of $\sim 30$ filters of width $\Delta \lambda / \lambda \sim 0.02$. A $S / N>20$ is generally necessary at the filters on the red side of the $H \alpha$ break to reach the target photometric accuracy. We estimate that a 5-year survey in a dedicated telescope with etendue in excess of $60 \mathrm{~m}^{2} \mathrm{deg}^{2}$ would be necessary to obtain a high enough density of galaxies to measure radial $\mathrm{BAO}$ with sufficiently low shot noise up to $z=0.85$. We conclude that spectroscopic surveys have a superior performance than photometric ones for measuring $\mathrm{BAO}$ in the radial direction.
\end{abstract}

\section{Introduction}

An important observable to constrain the nature of dark energy is the Hubble parameter $H(z)$ [1] , since it constitutes a more direct probe to the dark energy equation of state than the angular diameter distance $d_{a}(z)$ or the luminosity distance $d_{L}(z)$, which depend on an integral of $H(z)$. Einstein's equations imply that a homogeneous and isotropic universe, which is described by the FRW metric, that is composed of matter and dark energy with equation of state $p_{Q}=w_{Q}(z) \rho_{Q}$ expands according to

$$
\frac{H(z)}{H_{0}}=\left[\frac{\rho_{T}(z)}{\rho_{T}(0)}\right]^{1 / 2}=
$$




$$
\left[\Omega_{M}(1+z)^{3}+\Omega_{k}(1+z)^{2}+\Omega_{Q} \exp \left(3 \int_{0}^{z} \frac{1+w_{Q}\left(z^{\prime}\right)}{1+z^{\prime}} d z^{\prime}\right)\right]^{1 / 2},
$$

where the subscripts $Q, k, M$ and $T$ refer to dark energy, space curvature, matter, and total energy density, respectively. In the absence of space curvature, the quantities $d_{A}(z)$ and $d_{L}(z)$ are related to $H(z)$ via $d_{A}(z)(1+z)=d_{L}(z) /(1+z)=\int_{0}^{z} d z^{\prime} / H\left(z^{\prime}\right)$.

The acoustic oscillations in the photon-baryon plasma, observed as acoustic peaks in the CMB power spectrum, are also imprinted in the matter distribution at the scale of the sound horizon at the radiation drag epoch, when baryons were released from the photon pressure. The mass distribution is traced by the distribution of galaxies, and the Baryon Acoustic Oscillations (BAO) can be observed as a peak in the galaxy correlation function or as a series of harmonic oscillations in the galaxy power spectrum. The sound horizon at the radiation drag epoch can be computed very accurately from CMB observations (e.g., $r_{s}=153.2 \pm 2.0 \mathrm{Mpc}$ from WMAP5, 2]), so it provides a natural standard ruler. In fact, the galaxy power spectrum can be used to measure both the angular diameter distance through the clustering perpendicular to the line-of-sight, and the expansion rate $H(z)$ through the clustering along the lineof-sight. Therefore, BAO measurements test the relation between $H(z)$ and $d_{A}(z)$, providing constraints on dark energy and a limit to space curvature (e.g., 3, 4, 5, 6] ). The BAO technique is being considered a powerful probe to the nature of dark energy [7, 8, 9, 10, because of its potential to provide a standard ruler at different redshifts and its robustness to systematic effects.

For an ideal galaxy survey, [11 have shown that a volume of $1(\mathrm{Gpc} / \mathrm{h})^{3}$ at low redshift can constrain $H_{0}$ to the $7 \%$ level. Forthcoming surveys with larger volume are expected to reach the statistical power to constrain $H(z)$ at the $\%$ level. There are a number of requirements that these surveys need to satisfy for measuring BAO: covering a large survey volume, modeling the effects of galaxy bias and non-linearity, characterizing the covariance between different modes, evaluating the galaxy selection function to sufficient accuracy, reducing photometric calibration errors to low enough levels to avoid contamination of the BAO signal, etc. Measuring the BAO scale in the radial direction demands in addition that galaxy redshifts are measured to a sufficiently high accuracy, $\sigma_{z}$, to avoid an excessive smoothing of the BAO peak, which has an intrinsic width $\delta r \sim 10 \mathrm{Mpc}$, i.e., $\delta z \leq \delta r H(z) / c$. As shown by [12, a redshift accuracy $\sigma_{z}<0.003(1+z)$ is required to avoid substantial loss of accuracy of the $H(z)$ measurement from a given survey volume. Spectroscopic surveys usually yield a redshift accuracy much higher than this minimum requirement.

An alternative approach to measure the large number of redshifts required for $\mathrm{BAO}$ detection are photometric redshifts from imaging surveys. Broad-band photometry with $\sim 6$ filters usually reaches only to $\sigma_{z} /(1+z) \sim 0.03$ for the general population (with red galaxies having slightly smaller photo-z errors than blue ones), insufficient for measuring radial BAO. However, as the Combo-17 survey [13] has demonstrated, the galaxy photo-z accuracy can be improved by using a larger number of narrower filters. Photometric surveys can cover a large area of the sky faster than a spectroscopic survey and reach a higher number density of observed objects. We are therefore motivated to investigate the requirements for a photometric survey with medium to narrow bands to deliver interesting BAO measurements, and the optimization of the number of filters. Previous work has already explored this issue ([14, 12, 15, 16]). Here we concentrate specifically on the impact of the number of bands, signal-to-noise, and non-uniformity of the galaxy sample. We conclude with 
several considerations on the systematic effects that the photometric approach entails.

In our investigation we use both synthetic stellar population models and Sloan Digital Sky Survey (SDSS)-DR6 spectra of Luminous Red Galaxies. We concentrate on LRG because they have several properties that make them particularly useful for BAO surveys: they are a fairly homogeneous population, the form of their spectra makes them particularly suitable for good photo-z determinations, and their high luminosity facilitates reaching a high enough signal-to-noise up to high redshifts. For these reasons, LRG are the target of choice for $z<2$ BAO surveys (at higher redshift the Ly $\alpha$ forest probably provides the best method for measuring BAO; see [17]). The main conclusion we reach is that a spectroscopic survey is superior to narrow-band photometric surveys for measuring the radial BAO signal. We also show that if it were possible to find a very homogeneous population of LRG (with spectra closely matched by a single spectral template), then the photometric redshift accuracy would not substantially increase with the number of filters used beyond a total number of $\sim 10$ at a fixed total exposure time. This is not the case for galaxies measured at high signal-to-noise, for which a larger number of filters is optimal, but this high signalto-noise cannot be achieved for a large enough number of objects in a way that is competitive with the spectroscopic approach. In reality, however, the variability of realistic galaxy spectra worsens the photometric redshift accuracy, making it optimal to increase the number of filters to $\sim 30$ and requiring a higher signal-to-noise per filter to reach the desired redshift accuracy. These results are generally in good agreement with those of [15].

This paper is organized as follows: in $\S 2$ we review the requirements for a survey to measure radial BAO. The modeling of the LRG population is described in $\S 3$, and in $\S 4$ we describe our fiducial survey model. The results are presented in $\S 5$, where we analyze in detail the photo-z accuracy as a function of the number of filters, galaxy luminosity and redshift, first for ideal galaxies that match the templates precisely and then for real galaxies with SDSS spectra. Discussion and conclusions are presented in $\S 6$ and $\S 7$. Throughout this paper, we use a cosmological model with $H_{0}=70 \mathrm{~km} \mathrm{~s}^{-1}$ $\mathrm{Mpc}^{-1}, \Omega_{m}=0.3, \Omega_{\Lambda}=0.7$. Readers who want to quickly see the main conclusions or our study may wish to go directly to Figure 14 . This shows the number density of LRG in several redshift bins with photometric redshift better than $0.003(1+z)$ as a function of the etendue times the exposure time of a survey. It also shows the number density required to reach $n P=1$, necessary to make shot noise subdominant in the Fourier modes near the line-of-sight useful for measuring BAO.

\section{Spectroscopy vs photometry and target photometric requirements}

Future BAO surveys need to: a) cover large volumes of the universe sampling the acoustic scale $r_{s}=153.2 \pm 2 \mathrm{Mpc}$ with a density of galaxies high enough to make shot-noise subdominant, and b) not degrade with redshift errors the lineof-sight information that yields a measurement of $H(z)$. The latter condition implies, quantitatively, that the statistical photo-z errors need to be smaller than $\sigma_{z}=0.003(1+z)$ [12]. In Figure 1 we show the present-day $(\mathrm{z}=0)$ linear matter power spectrum (dotted line) and the corresponding line-of-sight redshift-space power spectrum (thin solid line), assuming a scale-independent bias $b=2$, a $\beta$ parameter $\beta=1 / b d \ln \delta / d \ln a \sim \Omega_{m}^{0.6} / b \simeq 0.24$, and assuming an intrinsic galaxy velocity dispersion of $420 \mathrm{~km} / \mathrm{s}$, corresponding to a pairwise velocity dispersion $\sigma_{p}=600 \mathrm{~km} / \mathrm{s}$, typical of non-linear redshift-space distortions. The line-of-sight power spectrum 


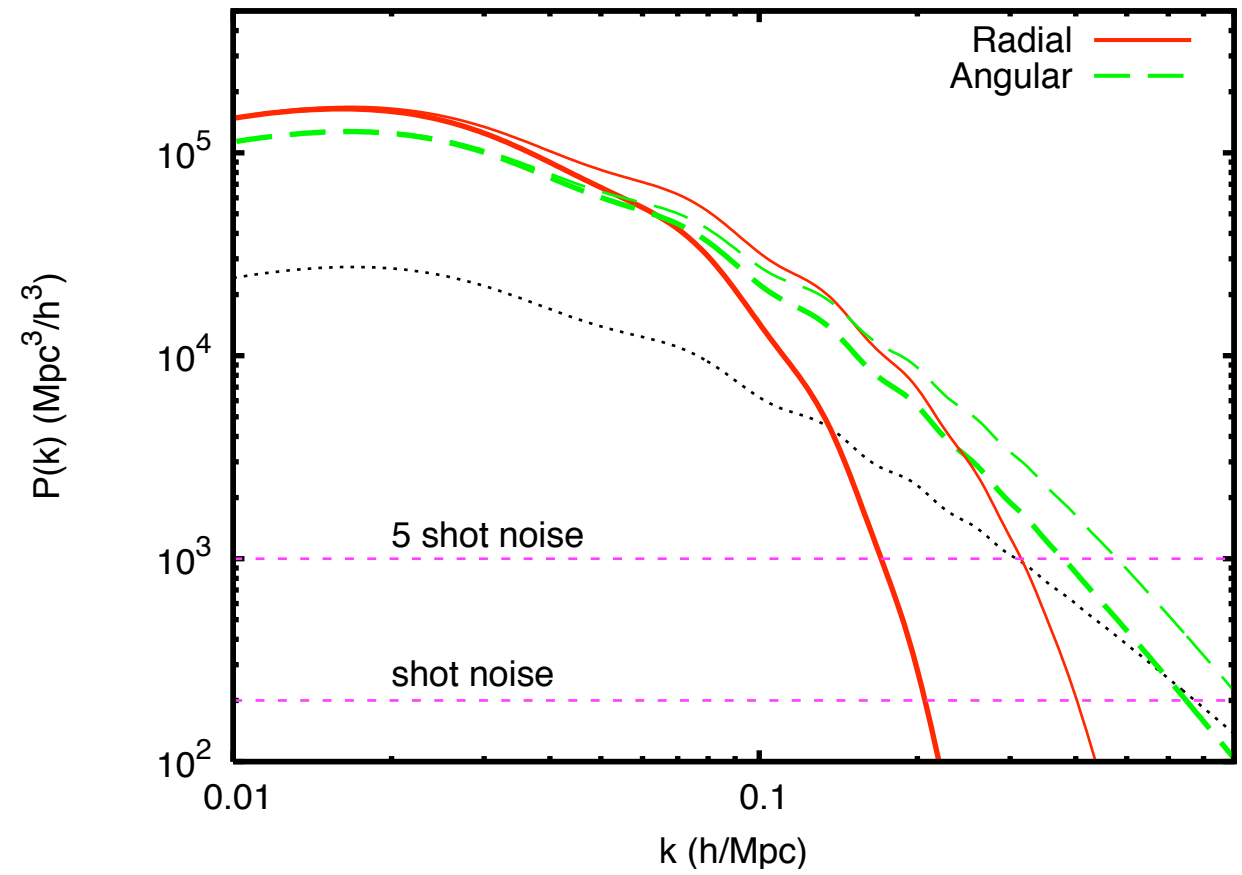

Figure 1. Galaxy power spectrum in the LCDM model. Dotted line: Presentday, real-space, linear matter power spectrum. Solid lines: Redshift space galaxy power spectrum on the line-of-sight, for a scale independent bias $b=2$ and redshift error $\sigma_{z}=0.003$ (thick line) or assuming typical small-scale redshift space distortions only (thin line). Long dashed lines: Angle-averaged power spectrum, also including redshift errors (thick) or not thin. The shot-noise contribution level for a galaxy density of $5 \times 10^{-3} h^{3} \mathrm{Mpc}^{-3}$ is shown by the short-dashed horizontal lines for reference.

is also shown with Gaussian photo-z errors of $\sigma_{z}=0.003(1+z)$ added (thick solid lines). The effects of biasing, linear redshift-space distortions [18, and the combined dispersion from photo-z errors and non-linear redshift-space distortions can be modelled as:

$$
P_{g}(k, \mu)=b^{2}\left(1+\mu^{2} \beta\right)^{2} P(k) D\left(k \sigma_{z} \mu\right),
$$

where $\mu=\cos \theta$, and $\theta$ is the angle with respect to the line-of-sight. In equation 2 the "Kaiser factor" is strictly valid only for linear, large-scale redshift-space distortions, but it will be sufficient for our main purpose of illustrating the requirements on the redshift accuracy and the shot-noise that need to be reached. The function $D\left(k \sigma_{z} \mu\right)$ describes the small-scale smearing of power. It is often parameterized by a Gaussian, $D\left(k \sigma_{z} \mu\right)=\exp \left[-\left(k \sigma_{z} \mu\right)^{2}\right]$, or by a Lorentzian, $D\left(k \sigma_{z} \mu\right)=\left[1+\left(k \sigma_{z} \mu\right)^{2}\right]^{-1}$. If $x \equiv k \sigma_{z} \mu<<1$, the two descriptions are equivalent since $\exp \left(-x^{2}\right) \simeq\left(1+x^{2}\right)^{-1}$. The effect of small-scale nonlinear redshift-space distortions is approximated as a contribution to $\sigma_{z}$ (subdominant in this case compared to redshift errors). Assuming $k \sigma_{z} \mu \ll 1$ and using the Lorentzian form for $D\left(k \sigma_{z} \mu\right)$, the angle-averaged (monopole) power spectrum can be calculated analytically with the following result:

$$
\int_{-1}^{1} P_{g}(k, \mu) d \mu \simeq
$$




$$
b^{2} P(k)\left[\frac{\left(\beta^{2}+6 \beta\right)}{3 k^{2} \sigma_{z}^{2}}-\frac{\beta^{4}}{k^{4} \sigma_{z}^{4}}+\left(\frac{1}{k \sigma_{z}}-\frac{\beta}{\sqrt{8} k^{3} \sigma_{z}^{3}}+\frac{\beta^{2}}{\sqrt{2} k^{5} \sigma_{z}^{5}}\right) \arctan \left(k \sigma_{z}\right)\right] .
$$

This monopole term is shown as the long-dashed line in Figure 1 . The Figure also shows the shot noise contribution (short-dashed lines) for a galaxy density of $\bar{n}=5 \times 10^{-3}$, and the level at which $\bar{n} P=5$ and $\bar{n} P=1$, which is where the shot noise contribution is negligible: in the line-of-sight, shot noise starts becoming comparable to the $P(k)$ signal at larger scales than for the angle-averaged $P(k)$.

Let us consider the following two cases: a photometric survey with target photo-z errors $\sigma_{z} \sim 0.003$ (thick lines), and a spectroscopic survey (thin lines, corresponding to unavoidable non-linear velocities). The sampling variance error on $P(k, \mu)$ scales as $\sigma_{P} / P=1 / \sqrt{2 \pi k^{2} \Delta k \Delta \mu V_{\text {eff }} /(2 \pi)^{3}}$, where $V_{\text {eff }}=$ $[\bar{n} P(k, \mu) /(\bar{n} P(k, \mu)+1)]^{2} V_{\text {survey }}$, and the accuracy at which the acoustic scale can be measured is directly proportional to $\sigma_{P} / P$. Thus, if the two surveys have the same number density of galaxies, and the spectroscopic survey reaches $n P=5$ along the line of sight at $k=0.32 \mathrm{~h} / \mathrm{Mpc}$, then the photometric survey reaches $n P=5$ at $k=0.16 h / \mathrm{Mpc}$. Note that the number of independent modes is roughly proportional to $k_{\max }^{3}$, and so the spectroscopic survey could obtain a much better constraint on the $\mathrm{BAO}$ scale from measuring the power on many more modes. competitive with a spectroscopic survey, a photometric survey would need to achieve a much higher galaxy density. If the survey volume were to be the same for the two surveys, then at $k=0.2 h / \mathrm{Mpc}$ a photometric survey would need a galaxy number density $\exp \left(-k^{2} \sigma_{z}^{2}\right) \sim 25$ times higher than a spectroscopic survey to achieve the same $\bar{n} P$ along the line of sight direction. The angle-averaged quantity is, of course, less sensitive to the smearing along the line of sight, and the same is true for all orientations where $\mu<1$. As shown by [12, at $\mathrm{z} \sim 1$ a redshift error of $0.3 \%$ degrades the error on $H(z)$ by a factor $\sim 2$, demanding therefore a survey with 4 times the volume of a spectroscopic survey to match its performance. As most forthcoming spectroscopic surveys will cover more than a quarter of the available sky (i.e., more than a quarter of the 30000 square degrees outside the galactic plane), the photometric approach might only be advantageous if it could reach higher redshifts.

The above considerations indicate that a spectroscopic survey is the favored option unless a much larger fraction of the sky can be covered with a photometric survey and with an extremely high object density; this is equivalent to imposing the requirement of $\sigma_{z}<0.3 \%$ down to fainter magnitudes. Below, we calculate if such a survey is possible. For this we concentrate on LRG, which are bright and have very homogeneous spectra, and we study the dependence of the results on intrinsic galaxy variability, luminosity, etc.

\section{Models for the Population of Luminous Red Galaxies}

We start by calculating the number density of LRG that can be observed at each luminosity and redshift. We use the [19] luminosity function of LRG and adopt their model of a Shechter luminosity function slope of $\alpha=-0.5$ (see Table 6 in [19]). To model the spectral distribution of galaxies, we use the first five templates presented in [20, §. These are empirical templates computed using the SPEED [21]

$\ddagger$ Since the relative error on the power spectrum $\sigma_{P} / P$ does not depend on redshift, the above considerations are valid at any redshift, with the caveat that the non-linearities decrease with $z$ but the photo-z errors do not.

$\S$ http://www.ice.csic.es/personal/jimenez/PHOTOZ/ 


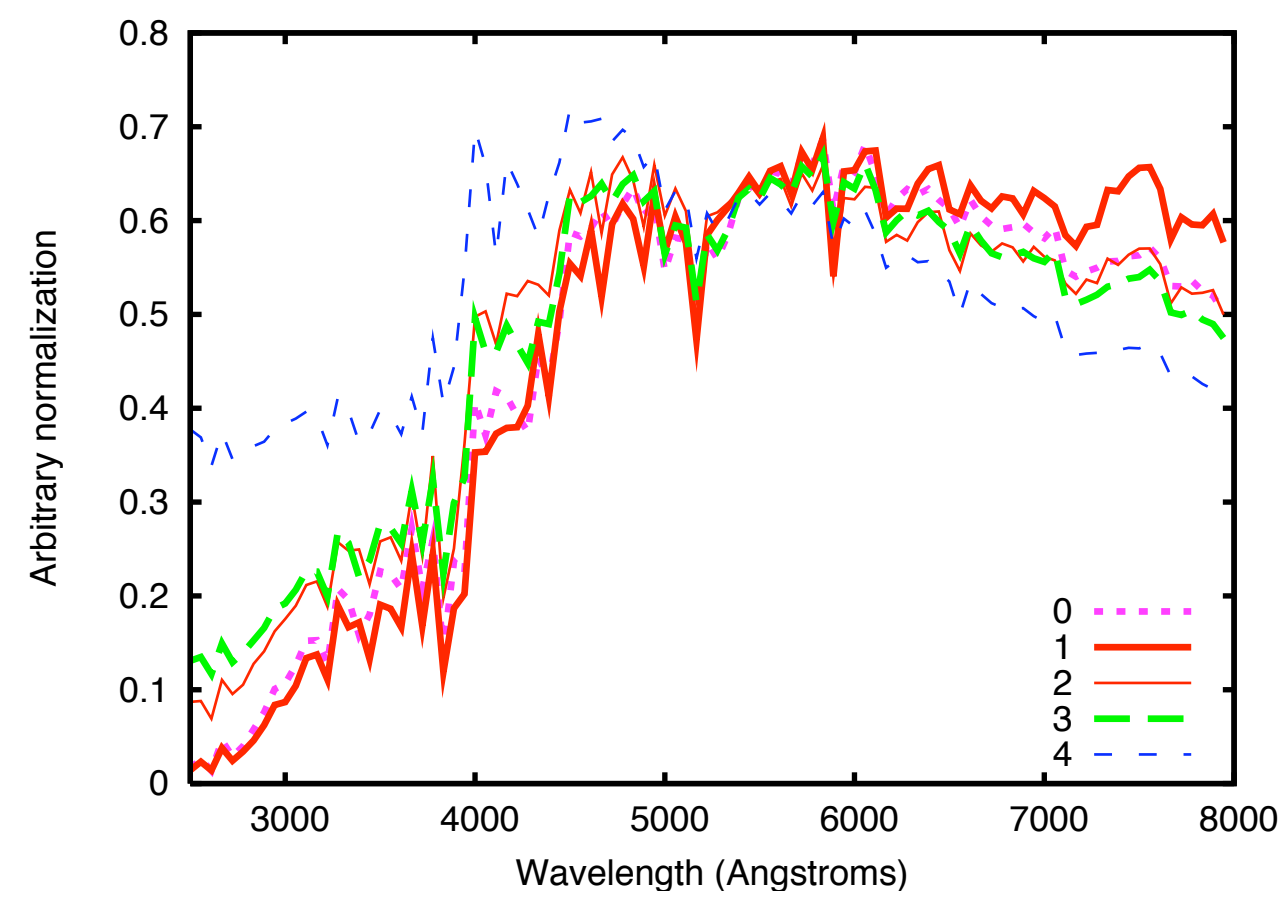

Figure 2. Spectral energy distribution of the five galaxy templates used in this paper, labelled from 0 to 4 , from [20]. For displaying purposes, in these figures a $20 \AA$ smoothing has been applied to the lines.

and CB08 (Charlot, private communication) models with solar metallicity and with star formation histories that are representative of the observed distribution in red galaxies over the stellar mass range $10^{9}$ to $10^{12} M_{\odot}$ extracted from [22]. The spectral templates correspond to the first five curves of Figure 4 in 22. The dust reddening is performed using the method described in 22 .

The spectra of the five templates, labeled by the numbers 0 to 4 , are shown in Figure 2 in the wavelength region of interest for this work. Template 0 is the spectrum of the oldest population and was build using the SPEED models, which have a different physical treatment for the giant and horizontal branches than the CB08 models. The average stellar population age is gradually reduced for the other templates, which use the CB08 models. We explain in the next section how we simulate the galaxy photometry using these spectral templates. We use the radius-luminosity relation measured in SDSS [23, 19],

$$
\log \left(r_{e}\right)=1.0-0.26\left(M_{B}-5 \log h+21.81\right)
$$

where the effective radius $r_{e}$ is expressed in $h^{-1} \mathrm{kpc}$ and $M_{B}$ is the B-band absolute magnitude. We include passive luminosity evolution (with fixed radius) as described by a 24 model where star formation starts at $z=4$ and decays with a timescale $\tau=0.6$ Gyr. This model provides a good match to the color evolution of LRG, as reported by [19]. A fixed aperture of $2 \operatorname{arcsec}^{2}$ will be assumed to simulate the galaxy photometry. This represents a reasonable compromise between minimizing the amount of galaxy light lost outside the aperture, and the noise contributed by the sky. Photometric redshifts are also most accurate when measured on fixed apertures, 


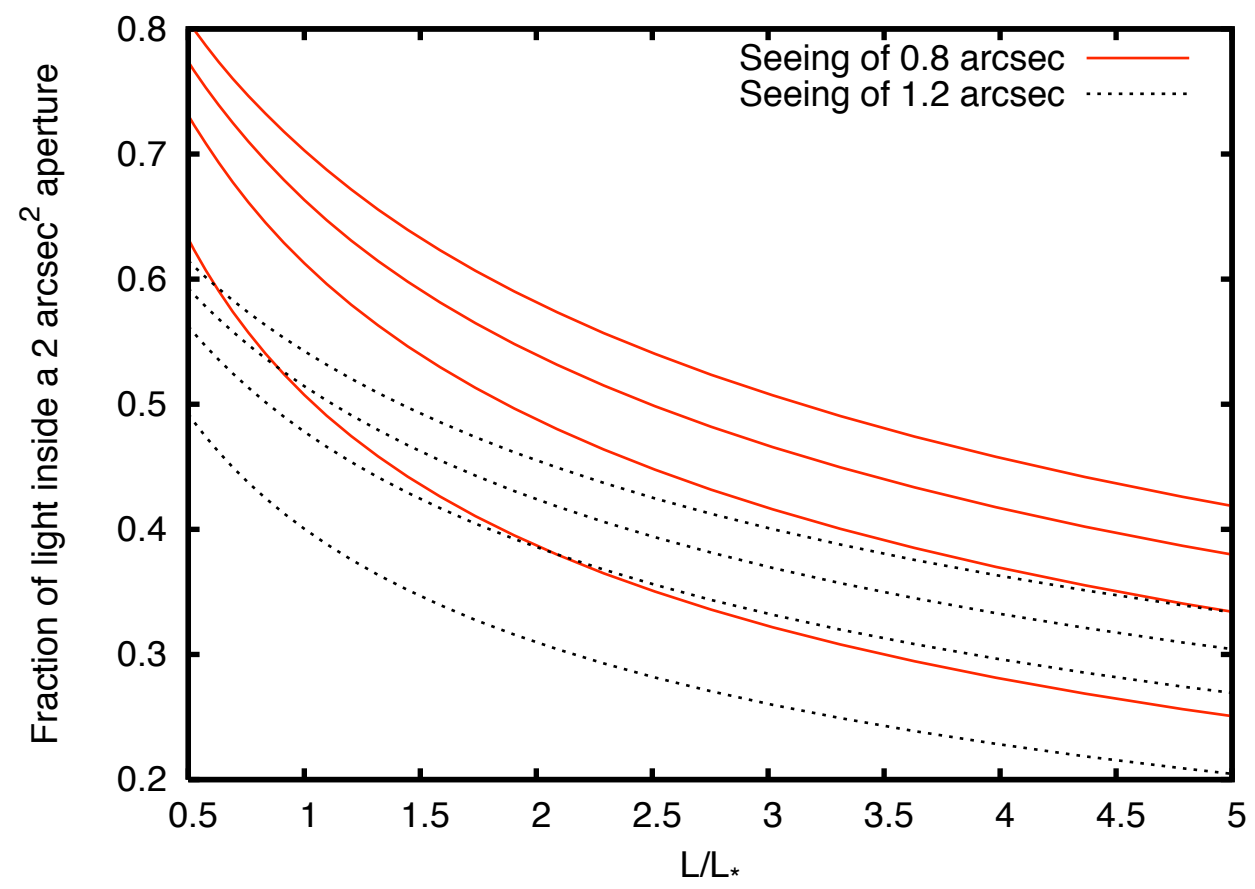

Figure 3. Fraction of light of a LRG within a $2 \operatorname{arcsec}^{2}$ aperture as a function of luminosity for four different values of redshift, $z=0.3,0.5,0.7,0.9$ from bottom to top, and two different values of the seeing, $0.8 \operatorname{arcsec}$ (solid lines) and $1.2 \operatorname{arcsec}$ (dotted lines).

rather than variable apertures that may be adjusted to the observed galaxy profile. We show in Figure 3 the fraction of galaxy light included within the aperture as a function of luminosity for four different values of redshift and two different seeings.

\section{Survey Model}

Throughout this paper we consider a fiducial narrow-band photometric survey as an example of the accuracy that can be achieved to measure a large number of LRG photometric redshifts, for the purpose of detecting BAO. We focus on narrow-band photometry in a wavelength range that is most useful for obtaining LRG redshifts over the range $z=0.5$ to $z=1.0$, using the $4000 \AA$ ( or $\mathrm{H} \alpha$ ) break, a blend of $\mathrm{H}$ and Ca lines. We choose a set of $N_{f}$ filters covering the fixed wavelength range from $\lambda_{1}=5300 \AA$ to $\lambda_{n}=8300 \AA$, dividing this range into $N_{f}$ intervals of equal wavelength width. We will consider different values $N_{f}$ to optimize this number for photometric redshifts for a given survey and telescope setup. The shape of the filter window functions is assumed to be a top-hat of width $\Delta \lambda=\left(\lambda_{n}-\lambda_{1}\right) /\left(N_{f}+1 / 2\right)$ || with the addition of lateral wings on each side where the window function varies linearly from zero to the value in the central top-hat. The width of each of the wings is set to $1 / 4$ of the width of the top-hat. The top-hat parts of the window function are adjacent and non-overlapping, whereas the wings cause the filters to have a certain degree of overlap. In a practical

\| The $1 / 2$ is due to the presence of the wings. 


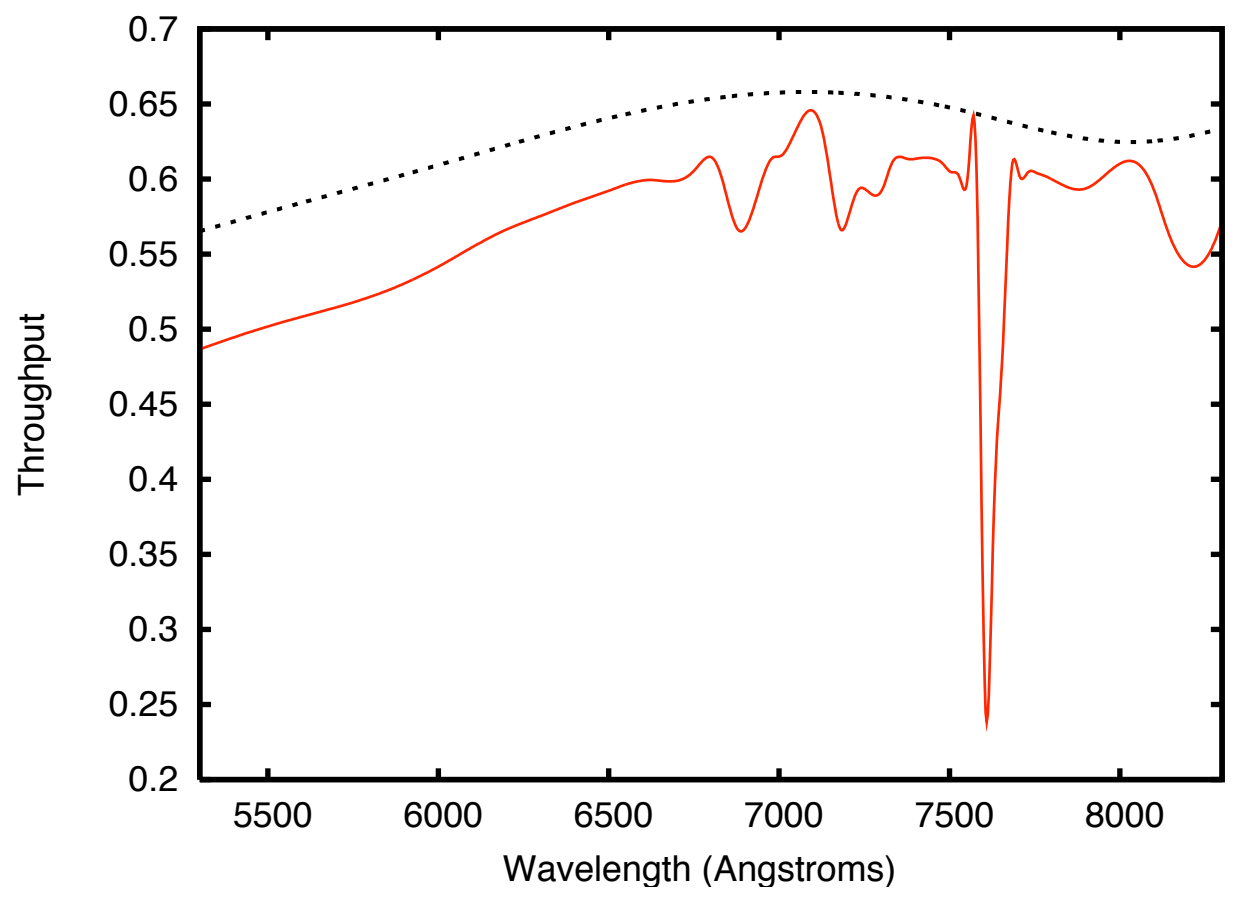

Figure 4. Overall throughput (solid) and throughput without including atmospheric absorption (dotted) used in this work.

application, it would probably be useful to complement this filter system with wider filters around the wavelength range considered here: this would eliminate some of the photo-z catastrophic failures (outliers in the photometric redshift error distribution). However, the addition of wide filters would not improve the accuracy of the good redshifts because they cannot contribute to a better resolution.

Figure 4 shows the overall throughput as a function of wavelength. We have included atmospheric absorption assuming an average of 1.2 atmospheric columns. We also include two mirror reflections, filter transmission and CCD efficiency from [26, 27, 28]. We use the sky brightness of Patat (2008, private communication; 25]), measured for the Paranal Observatory, to simulate the photometric noise in each band. This is adequate for a dark site in the absence of any artificial light, and a mean airglow intensity over the solar cycle. Figure 5 shows the spectrum of the sky in the AB magnitude system, and the average sky brightness in each one of the filters for the case $N_{f}=30$ (blue diamonds). The squares and circles indicate the noise levels in our fiducial survey and are discussed below.

Once the shape of the filters, the overall throughput, sky brightness, source flux and radius, and seeing have been fixed, the photometric precision reached by a survey for the flux in each filter is proportional to $\left[E t /\left(N_{f} \Omega\right)\right]^{1 / 2}$, where $E$ is the etendue (the product of the effective telescope aperture times the field of view), $t$ is the total survey observing time, and $\Omega$ is the total solid angle covered by the survey. We shall assume for our fiducial survey a product $E t=1.5 \times 10^{5} \mathrm{~m}^{2} \mathrm{deg}^{2} \mathrm{hr}$, corresponding for example to a characteristic case of a dedicated telescope with etendue $E=30$ (e.g., a $3 \mathrm{~m}$ telescope with a 5 square degrees field of view) that can obtain good 


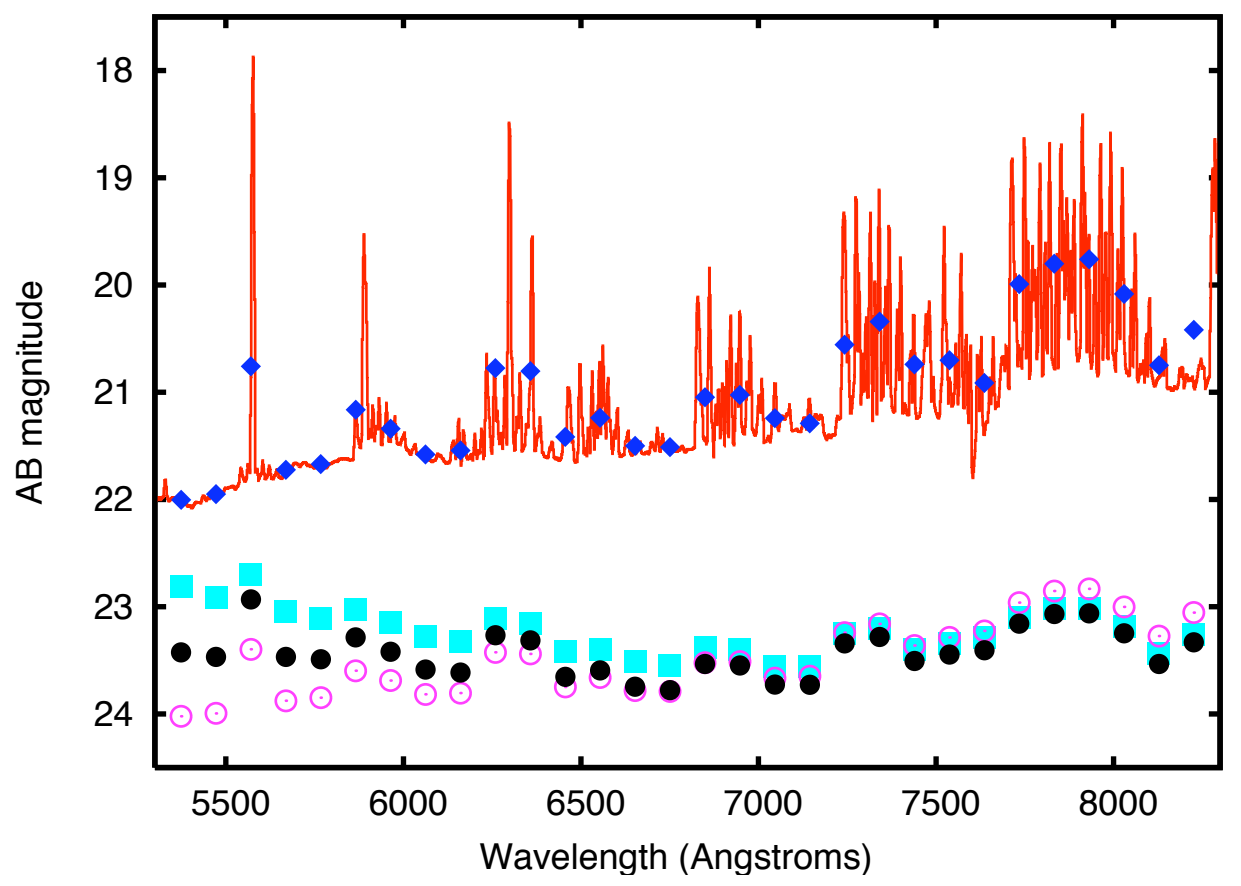

Figure 5. Solid line: Paranal sky spectrum from 25. Diamonds: Sky surface brightness in $\mathrm{AB}$ mag/ $/ \operatorname{arcsec}^{2}$ in 30 equally-spaced narrow-band filters. Empty circles: $5 \sigma$ sky noise for an aperture of $2 \operatorname{arcsec}^{2}$ in 30 narrow-band filters using a constant exposure time in our fiducial survey. Full circles: $5 \sigma$ sky noise for an aperture of $2 \operatorname{arcsec}^{2}$ in 30 narrow-band filters using a linearly growing exposure time, with five times longer exposure in the reddest filter than in the bluest one. Squares: $5 \sigma$ magitude limits, with the same exposure times as for the full circles.

photometric data for 1000 effective hours of observation per year, over a period of 5 years. We assume $\Omega=8000$ square degrees, close to the maximum area observable from a non-equatorial site, since, in general, the best strategy for measuring $\mathrm{BAO}$ is to cover the widest possible area. We also assume an optimistic value for the seeing of $0.8^{\prime \prime}$, corresponding to the best observatories in the world. Figure 3 can be used to see how the signal-to-noise is degraded for larger seeing: the required value of $E t$ to reach a fixed signal-to-noise scales as the inverse square of the fraction of light within the aperture. For example, a change of the seeing from $0.8^{\prime \prime}$ to $1.0^{\prime \prime}$ corresponds to a $10 \%$ reduction in the fraction of light within the aperture, which must be compensated by an increase of $20 \%$ in $E t$.

The number of useful observing hours per year obviously depends on the quality of the observing site. The total number of night hours per year is $\sim 3500$; the number of observed hours is reduced owing to cloudy or non-photometric (i.e., affected by cirri) nights, and Moon time. Ideally, the presence of increased sky brightness (either artificial or lunar) simply means that the effective observed time is reduced as the inverse of the sky brightness, because the photometric noise is inversely proportional to the square root of the ratio of the sky brightness over the exposure time, when the noise is dominated by the sky. For example, an increase by half a magnitude of the sky brightness needs to be compensated by an increase of $E t$ by a factor $10^{0.2} \simeq 1.6$. 
This implies an effective reduction of the observed time due to the Moon of $\sim 25 \%$, averaged over the Moon cycle, in the wavelength range of interest. Our assumption of 1000 effective hours of observation per year therefore assumes that $\sim 40 \%$ of the night time is clear and with acceptable photometric conditions. The need to co-add images obtained under different seeing, sky brightness and transparency conditions also needs to be taken into account as an added difficulty. In order to do faint galaxy photometry, one often needs to discard the images with the worst seeing and convolve the rest to a common maximum seeing, in order to avoid systematic photometric errors that depend on galaxy morphology and may tend to introduce artificial correlations in the photometric redshift errors. The assumptions we make here should therefore be regarded as optimistic and corresponding to an excellent observing site.

For our chosen value of $E t=1.5 \times 10^{5}$, and seeing of $0.8^{\prime \prime}$, Figure 5 shows the $5-\sigma$ sky noise in $\mathrm{AB}$ magnitudes within a fixed aperture of $2 \operatorname{arcsec}^{2}$. The open circles show the sky noise when the total exposure time is divided equally among $N_{f}=30$ filters, and the filled circles assume the exposure time is a linearly increasing function of wavelength to reduce the noise in the reddest filters and achieve a more uniform LRG redshift accuracy over the range $0.5<z<0.9$. For the latter case, we set the exposure time for the reddest filter at 5 times that for the bluest one. We have found these distribution of exposure times to be a reasonable compromise to achieve the best redshift accuracies for the largest number of galaxies. We shall use these variable exposure times for our fiducial survey throughout the paper, with 5- $\sigma$ sky noise indicated by the filled circles. To compute the total noise in our simulated photometry, we add quadratically the sky, source and read-out noise. The read-out noise in a photometric mesurement depends on the number of exposures, the pixel size and the quality of the CCDs. We model the read-out noise as 7 electrons per pixel at each exposure, with a pixel size of 0.4 arcsec. We assume that three exposures are obtained for each field and filter (in practice the number of exposures could not be reduced below 3 in most filters if the telescope operates in passive drift-scanning mode, the best strategy to minimize photometric calibration errors). The magnitude of a source detected at a signal-to-noise of 5 under these assumptions is shown as the filled squares in Figure 5. The squares approach the filled circles (the 5- $\sigma$ sky noise) as the read-out and source noise become small compared to the sky noise.

The 1- $\sigma$ sky noise is shown in Figure 6 as filled circles. The curves show the AB magnitudes of an $L_{*}$ galaxy in each of the $N_{f}=30$ filters, at four different redshifts. Note that the true $\mathrm{S} / \mathrm{N}$ measured for an $L_{*}$ galaxy is slightly decreased compared to that shown in this Figure because of the source photons contribution to the total noise; this is only a $\sim 10 \%$ effect for an $L_{*}$ galaxy at $z=0.5$ on the first filter to the red of the $H \alpha$ break.

To model the accuracy of photometric redshifts, we proceed as follows in the next section: first, we simulate the $N_{f}$-filter photometry of a galaxy that has the spectrum of one of the five templates we use. We add noise for a survey with the characteristics described above, and fit the resulting magnitudes with the same template. This will tell us the minimum photometric redshift error that would be obtained in the ideal case of a galaxy for which we know exactly the spectral shape, and we fit only one parameter, the redshift, to the measured photometry. We refer to this as "single template case". We shall examine the dependence of the results on the template used, and comment on the effects of galaxy evolution. We shall then consider the case where a linear combination of the five templates is used to fit to the photometry simulated with one template (5-templates case) and the case where the full set of 5 templates is 


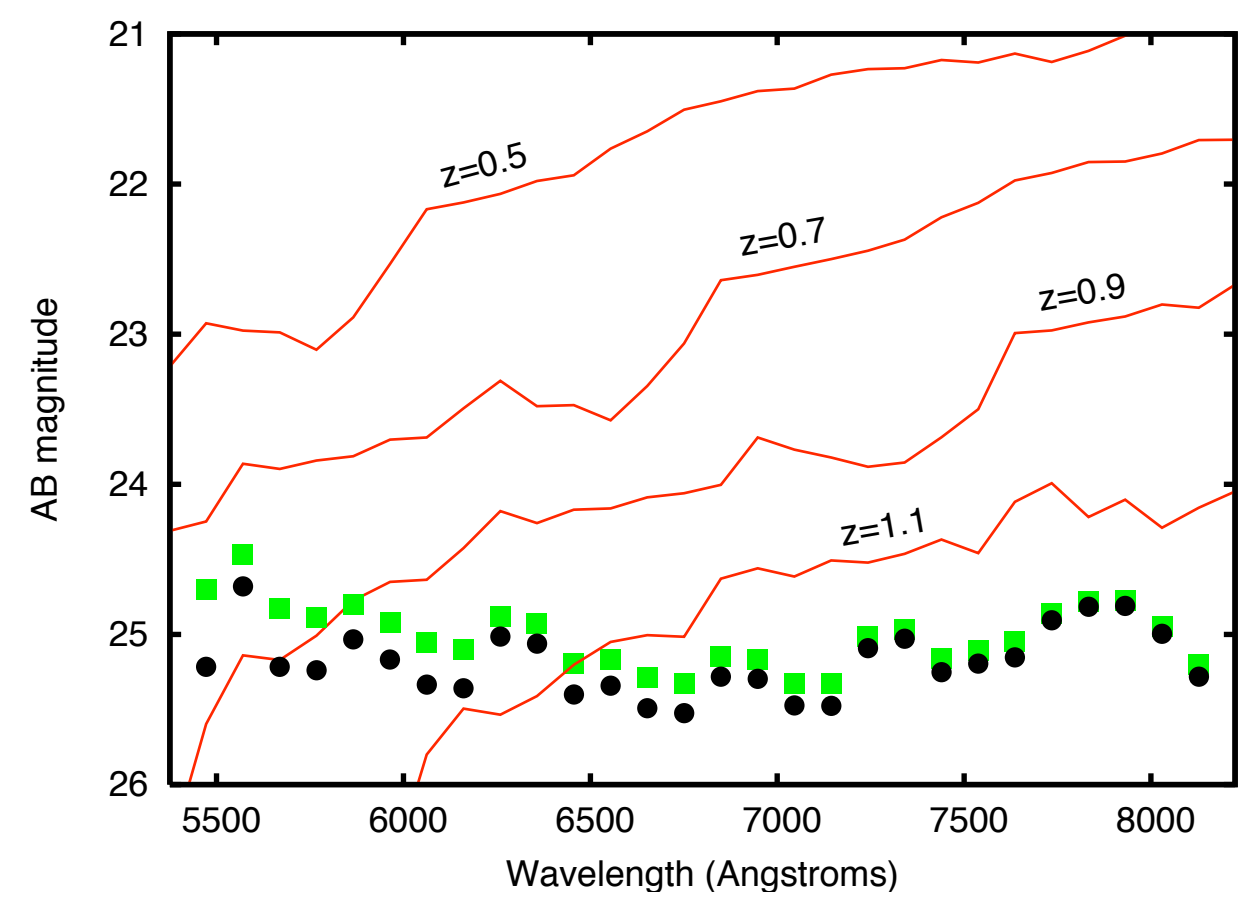

Figure 6. Simulated photometry of $L_{*}$ galaxies using our template 1 at $z=0.5,0.7,0.9,1.1$ (solid lines), and the $1 \sigma$ sky noise (circles) and magnitude limits (squares).

used to simulate and fit the photometry (5-to-5 templates case). This will show how the redshift error increases when galaxies are allowed to have variable spectra with five parameters (the redshift and four relative template amplitudes), but the spectra are still assumed to be precisely modelled by the templates used in the fit. In these two cases, the effect of changing the number of filters will be explored.

Finally, we shall use observed spectra of LRG from the SDSS public data to generate a photometric "mock" catalog, and fit their photo-z's using our 5 templates. The resulting photometric errors should be more realistically expected for real galaxies, which are not perfectly matched by a limited number of templates. We note that the 5 templates we use have been optimized to fit the spectra of a wide class of observed galaxies, and therefore they should be an ideal set for obtaining the best possible photometric redshifts.

\section{Photometric Redshift Accuracy}

\subsection{Optimal number of filters: analytic considerations}

Before presenting our numerical results on the simulated photometry described in the previous sections, it will be useful to derive some analytical results on the photo-z errors and the optimization of the number of filters under some simplifying approximations, using simple examples of galaxy spectra. We consider here the ideal case where a galaxy has exactly the same spectrum as the template used to fit the 
measured photometry, and approximate the filter windows as a top-hat.

If a fixed total exposure time is to be divided among all the filters, then the signalto-noise in each individual filter, $F / \sigma_{F}$ (where $F$ denotes flux and $\sigma_{F}$ its statistical error), is inversely proportional to the number of filters $N_{f}$ : the exposure time in each filter is $t_{f} \propto N_{f}^{-1}$, and the wavelength width of each filter is $\Delta \lambda \propto N_{f}^{-1}$. Hence, the number of photons detected in each filter is $N_{p h} \propto t_{f} \Delta \lambda \propto N_{f}^{-2}$, and $F / \sigma_{F} \propto N_{p h}^{1 / 2} \propto N_{f}^{-1}$. As we increase the number of filters, the resolution increases at the expense of the achieved signal-to-noise.

To model the use of the $\mathrm{H} \alpha$ break in LRG, we first consider a galaxy spectrum that has a break at wavelength $\lambda_{0}$, where the flux per unit wavelength is $F_{\lambda}=F_{0}$ at $\lambda>\lambda_{0}$ and $F_{\lambda}=(1-B) F_{0}$ at $\lambda<\lambda_{0}$. The filter that includes the break wavelength $\lambda_{0}$ has a flux

$$
F=F_{0}(1-B x),
$$

where $x=\left(\lambda_{i}-\lambda_{0}\right) / \Delta \lambda$, and $\lambda_{i}$ is the wavelength of the right edge of the filter. From the measured value of $F$ (and assuming that we know exactly the values of $F_{0}$ and $B$ from the measurements in the other filters), the wavelength $\lambda_{0}$ can be measured to an accuracy

$$
\sigma_{\lambda}=\Delta \lambda \frac{\sigma_{F}}{F_{0} B} .
$$

Since $\sigma_{F} / F_{0} \propto N_{f}$, and $\Delta \lambda \propto N_{f}^{-1}$, we conclude that the error to which the wavelength $\lambda_{0}$ is measured is independent of the number of filters, and results in a photometric redshift accuracy $\sigma_{z}$ given by

$$
\frac{\sigma_{z}}{1+z} \equiv \sigma_{l z}=\frac{\sigma_{\lambda}}{\lambda}=\frac{\Delta \lambda}{\lambda} \frac{\sigma_{F}}{F_{0} B} .
$$

In other words, unresolved breaks in the spectra of galaxies yield a redshift measurement that does not improve as the number of filters is increased. This is correct only in the idealized situation where the amplitude of the break is perfectly known and galaxy variability may be ignored.

For a resolved break, we assume that the flux varies linearly from $F_{0}$ to $F_{0}(1-B)$ over a wavelength range $\lambda_{0}$ to $\lambda_{0}+\delta \lambda$. The flux measured in a filter centered at wavelength $\lambda_{i}$ and which is fully included within the interval of the break of width $\delta \lambda$ is $F=F_{0}(1-B x)$, where $x=\left(\lambda_{i}-\lambda_{0}\right) / \delta \lambda$. Hence, the error on $\lambda_{0}$ that can be deduced from the flux measured in one filter only is

$$
\sigma_{\lambda}=\delta \lambda \frac{\sigma_{F}}{F_{0} B} \propto N_{f}
$$

Since the number of filters contained in the wavelength range of the resolved break, $\delta \lambda$, is proportional to $N_{f}$, and the combined error from the measurement in all the filters is reduced as $N_{f}^{-1 / 2}$, we find that the set of all photometric measurements will yield an error $\sigma_{\lambda} \propto N_{f}^{1 / 2}$. Therefore, the filters should not be any narrower than the intrinsic width of any breaks that substantially contribute to the photometric redshift measurement.

On the other hand, if the spectrum contains features similar to an emission or absorption line, then it is advantageous to increase the number of filters. For a line feature with an equivalent width $W_{\lambda}$ which is entirely contained in one filter, the measured flux is $F=F_{0}\left(1+W_{\lambda} / \Delta \lambda\right)$, independently of the position of the line within a top-hat filter. The difference $F-F_{0}=F_{0} W_{\lambda} / \Delta \lambda \propto N_{f}$ can be measured to an 
accuracy $\sigma_{F} \propto N_{f}$, so the position of the feature is measured to increasing accuracy as the number of filters is increased, up to the point where the feature is resolved.

In practice, the spectra of LRG contain several features that may be approximately modelled as breaks and/or line features of different wavelength widths. In the case of a single template, the optimal number of filters results from the contribution of all the features to the determination of the photometric redshift. At the same time, the spectra of real galaxies are, of course, not described by a single template and depend on a variety of parameters. The need to fit for these spectral variations in addition to the redshift favors an increased number of filters when the signal-to-noise that can be reached is high enough. This is seen in more detail in the following section; the analytic considerations discussed above will be helpful to interpret the numerical results for fits to simulated photometry of LRG.

\subsection{Single template galaxies}

We now present the numerical results of recovering photometric redshifts from the simulated photometry of LRG spectra, for the case of our fiducial survey as described in $\S 4$. The distribution of photometric redshift errors is generally not Gaussian because of the presence of outliers, or catastrophic failures, which naturally increase as the signal-to-noise drops. To avoid having to deal with identification of outliers, we report the photo-z error $\sigma_{z}$ in all of our figures, unless otherwise stated, as the interval such that $68 \%$ of the simulated galaxies have a smaller difference between their true redshift and fitted redshift. As long as the fraction of outliers is small, the 68 percentile value should approximately correspond to the rms redshift error after the outliers have been successfully removed. We shall not discuss here the methods for removing outliers in photometric redshifts; these would depend on how our assumed narrow-band filter system is complemented with wider filters on a broader range of wavelengths. In practice, any method for fitting photometric redshifts will fail to detect some outliers and will classify some good redshifts as outliers. Our assumption that outliers are removed perfectly is the most optimistic possible one.

We start with the simplest case where the same template used to generate the galaxy photometry is then used to fit the photometric redshift. This corresponds to the ideal case where the galaxy spectral shape is precisely known, and only the redshift and luminosity need to be fitted. We use template 1 for this purpose in this section. The relative redshift error $\sigma_{l z}=\sigma_{z} /(1+z)$ is shown in Figure 7 (top panel) as a function of redshift, for galaxies of luminosity $L_{*}$ and varying the number of filters. For this Figure, we show both the 68 percentile error (thick lines) and the 95 percentile error (thin lines). The thin and thick lines should be separated by a factor 2 for a Gaussian distribution of errors, and by an increasing factor as the fraction of outliers increases. The redshift errors are calculated at 450 values of the redshift, from $z=0.1$ to $z=1$ in increments of $\delta z=0.002$. For each value of the redshift, the error is computed from a total of 100 photometry simulations. Therefore, each curve in Figure 7 (top panel) is based on a total of 45000 simulations of galaxy photometry. The results are shown as filled dots, after averaging in quadrature over redshift intervals of width $\Delta z=0.1$ (lines between dots are plotted for guidance only). The bottom panel of Figure 7 shows the redshift error (68 percentile) as a function of galaxy luminosity for redshifts $0.65,0.75$ and 0.85 (with errors increasing with redshift), again after averaging over an interval $\Delta z=0.1$, and for $N_{f}=10,30$ and 50 .

We see from this Figure that for galaxies of luminosity $L_{*}$, the redshift errors are 

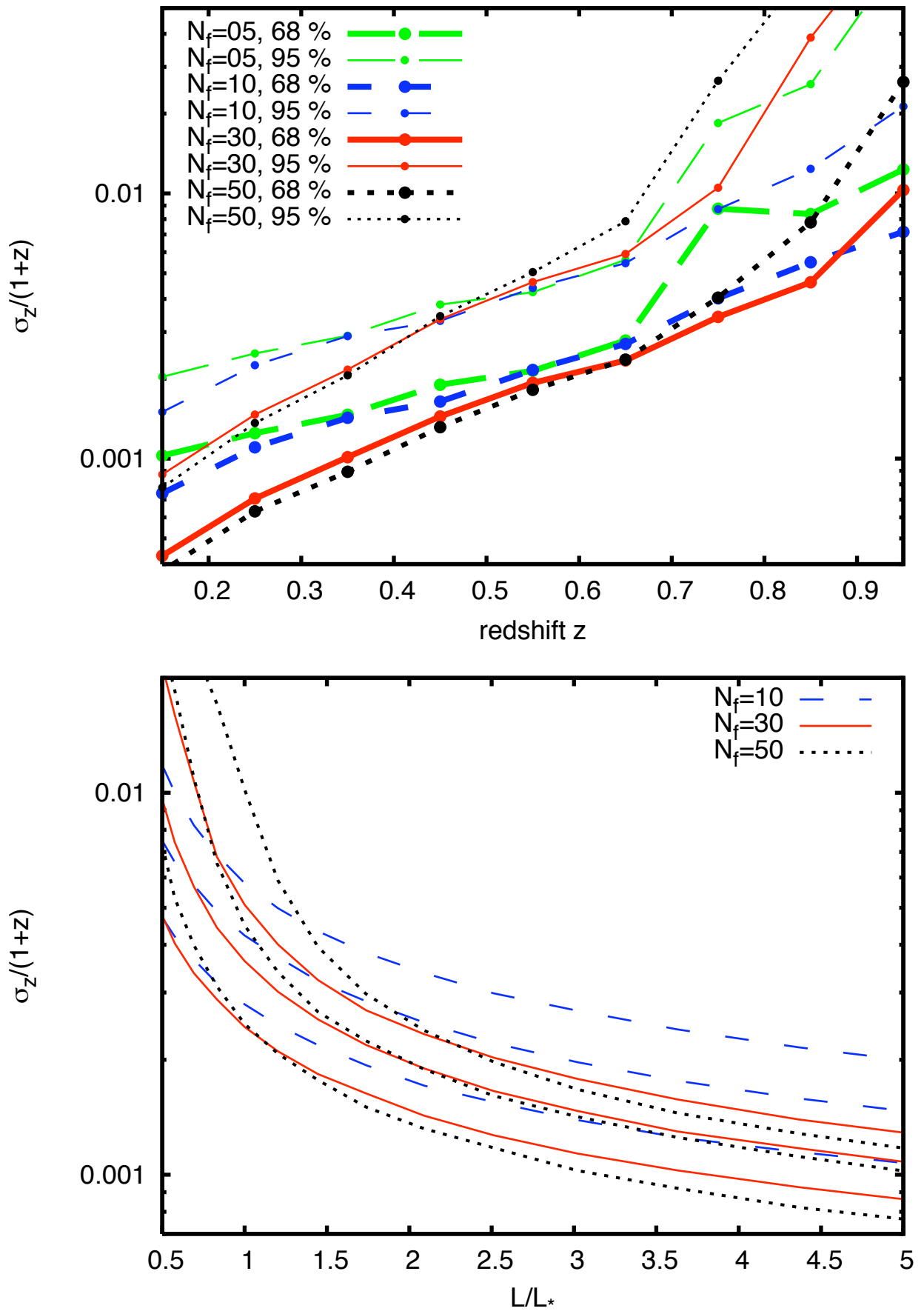

Figure 7. Top: Photo-z error as a function of redshift of an $L_{*}$ galaxy in our fiducial survey, varying the number of filters as indicated. The 68 or 95 percentile error is computed in each redshift bin of width $\Delta z=0.002$, and then averaged in quadrature over $\Delta z=0.1$. Bottom: Photo-z error (68 percentile, computed as in the top panel) as a function of galaxy luminosity, for redshift intervals centered at $z=0.65,0.75$ and 0.85 (errors increase with redshift), and for $N_{f}=10,30$ and 50 . Template 1 is used in all cases for generating and fitting the galaxy photometry. 
practically independent of the number of filters in our fiducial survey. At $z>0.4$, there is only a small improvement of $\sigma_{z}$ when $N_{f}$ is increased from 10 to 30 , and even this small improvement is offset by an increasing fraction of outliers as $N_{f}$ is increased. This implies that it would not be worth to use more than $\sim 10$ filters to measure photometric redshifts for galaxies of luminosities near $L_{*}$ that are well fitted by a single template.

The target accuracy of $0.003(1+z)$ to measure radial $\mathrm{BAO}$ is achieved up to $z \sim 0.7$ for $L=L_{*}$. The bottom panel shows that at $z=0.9$ the same target can be achieved for $L>1.6 L_{*}$. For galaxies of high luminosity, increasing $N_{f}$ from 10 to 30 becomes a stronger advantage, but gains for $N_{f}>30$ are not substantial, especially because the outliers increase with $N_{f}$. The approximately constant redshift accuracy with the number of filters is roughly a consequence of the analytic arguments discussed in $\S 5.1$ for the case when the main spectral feature is an unresolved break. Note that there are also numerous features in the spectrum of template 1 (Figure 2) that can be modelled as absorption lines of width $\sim \lambda / 30$ (particularly at wavelengths just blueward of the $\mathrm{H} \alpha$ break), which are the principal reason for the improvement of redshift errors from $N_{f}=10$ to $N_{f}=30$ for high signal-to-noise.

So far, the results have been shown averaged over redshift bins of $\Delta z=0.1$. The unbinned results are shown in Figure 8, for the cases $N_{f}=10$ and $N_{f}=50$. There are periodic oscillations in the redshift error, with a spacing $\delta z$ that corresponds to the separation between filters. These oscillations are due to different features in the spectrum (mostly the $\mathrm{H} \alpha$ break) crossing the center and the edge of filters as the redshift varies. The redshift error is best (smallest) when the $\mathrm{H} \alpha$ break is placed between two filters, and it is worst when it is placed at the center of one filter. These oscillations should naturally depend on the shape of the filter windows, as well as the form of the $\mathrm{H} \alpha$ break, and therefore the type of LRG galaxy. One should note that the scale of the oscillation corresponds to the BAO scale for $N_{f} \simeq 30$. The presence of these artificial oscillations in the redshift error, which could vary their amplitude over the survey area owing to variations of the seeing or other observing conditions that may systematically affect the photometry, needs to be properly calibrated and corrected for in order to avoid introducing perturbations in the $\mathrm{BAO}$ peak of the galaxy correlation function.

\subsection{Using multiple templates}

In reality LRG are not a perfectly uniform population described by a single spectrum. We now see the impact on the redshift errors when variations in the galaxy spectra are allowed for. We use the five templates described in $\S 3$, which have been optimized to provide a good representation of observed galaxy spectra [20, 22.

Figure 9 shows the photo-z error for spectra generated with template 1 and $L=L_{*}$. The thin lines are the same as those shown in Figure 7 (top panel), fitted with the same template 1 . The thick lines are the result obtained when any linear combination of the five templates is allowed in the fit. As expected, the errors increase when we allow for spectral variability: the maximum photo-z accuracy for an ideal galaxy having exactly the same spectrum as template 1 is obtained when we know that the galaxy has the spectrum of template 1 . When we do not know this, and spectral variability needs to be allowed for, the photo-z error is larger. When fitting with our five templates, the target accuracy of $\sigma_{z}=0.003(1+z)$ is achieved only for $z<0.63$ at $L=L_{*}$ for $N_{f}=30$ (which is roughly the ideal number of filters). Results 

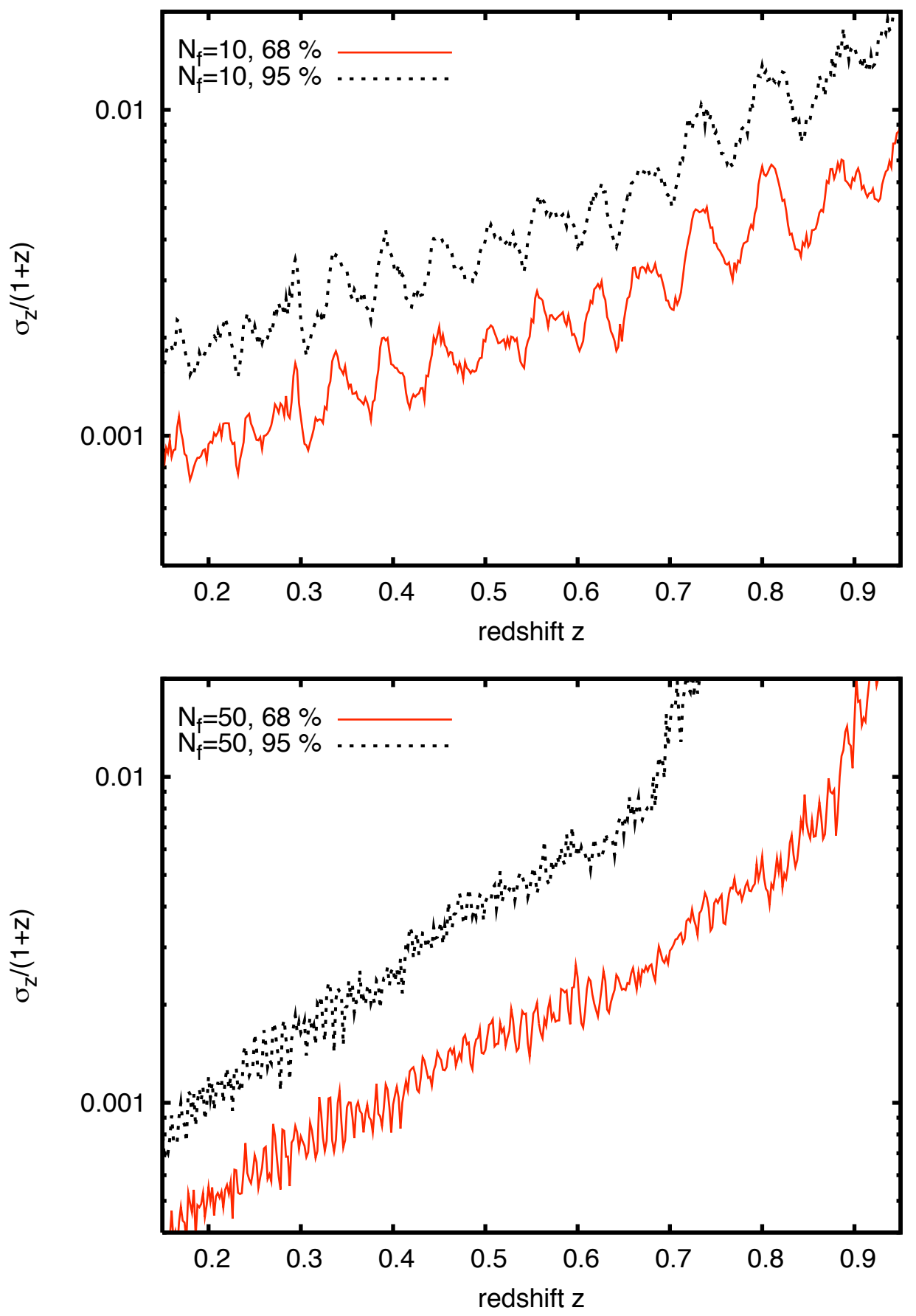

Figure 8. Photo-z error as a function of redshift without smoothing for 10 filters (top) and 50 filters (bottom). The oscillations have a periodicity determined by the filter width. 


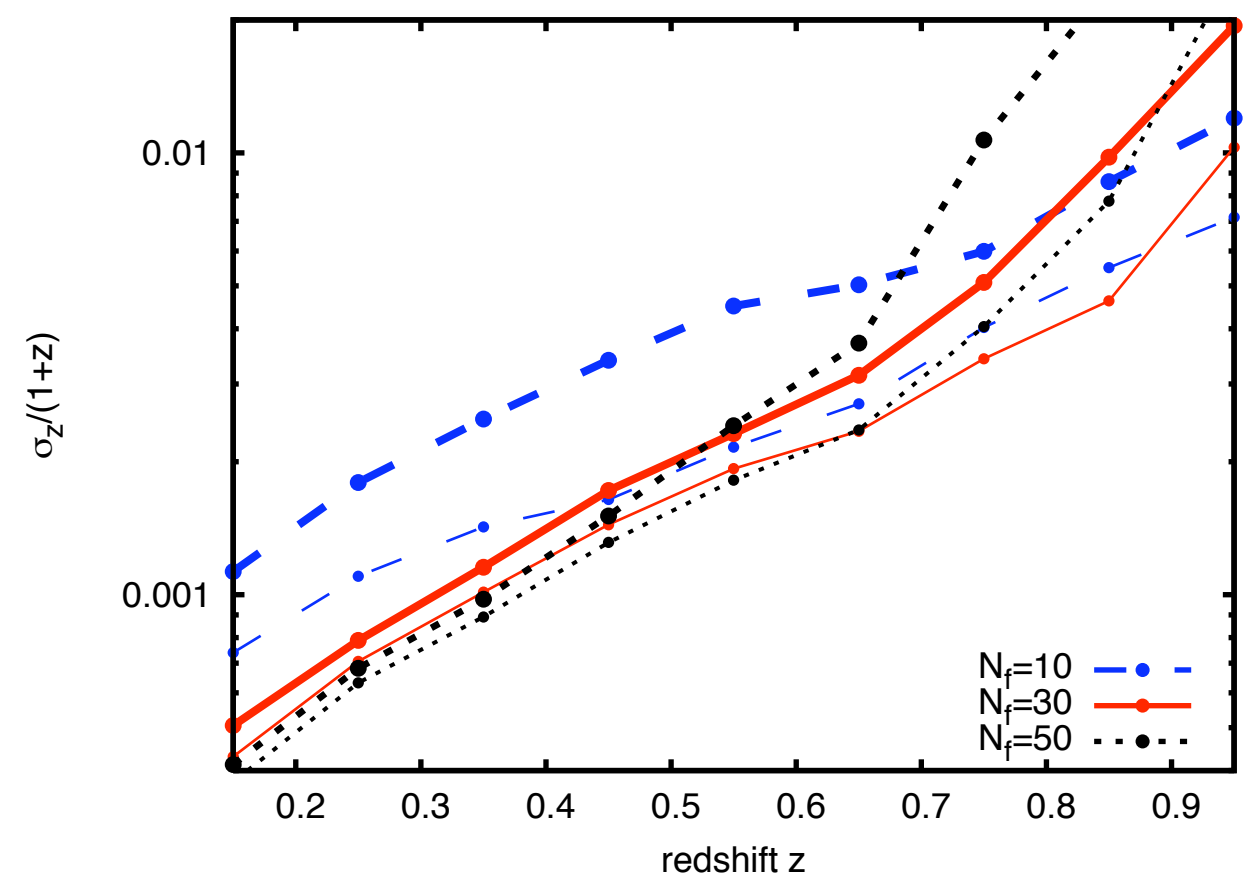

Figure 9. Impact of using multiple templates on redshift errors. Thin lines: The mock $L_{*}$ galaxy photometry is generated using template 1 and fitted with the same template. Thick lines: The same mock catalogs generated with template 1 are fitted with all five templates. The 68 percentile errors are computed as in Figure 7 The number of filters is varied as indicated.

for $L>L_{*}$ will be shown below in Figure 13 .

The increase of the redshift error resulting from fitting with five templates is substantially worse for $N_{f}=10$. When LRG are not assumed to be a homogeneous population, using a larger number of filters becomes more advantageous because of the need to distinguish the type of galaxy spectrum when fitting the redshift. However, as found in the previous section, increasing further the number of filters to $N_{f}=50$ is generally not worthy, except at rather high signal-to-noise, where the number density of sources is small in our fiducial survey.

The results in Figure 9 are a lower limit to the increase of redshift errors caused by galaxy variability, because real galaxies are not perfectly matched by the set of five templates we use. We discuss this further in $§ 5.5$.

\subsection{Variation of redshift error with templates and effects of galaxy evolution}

Figure 10 (top panel) shows the photo-z errors when the galaxy photometry is generated for each one of the five templates, and fitted allowing a linear combination of all five templates, for $L=L_{*}$ and $N_{f}=30$. There are substantial variations of the redshift error with the template spectrum, implying that the redshift error depends on the type of LRG galaxies selected to measure BAO. Over the redshift range $0.5<z<0.9$, which is the most promising for our purpose, template 0 gives the smallest errors and templates 2 and 4 the largest ones. The long-dashed line shows 

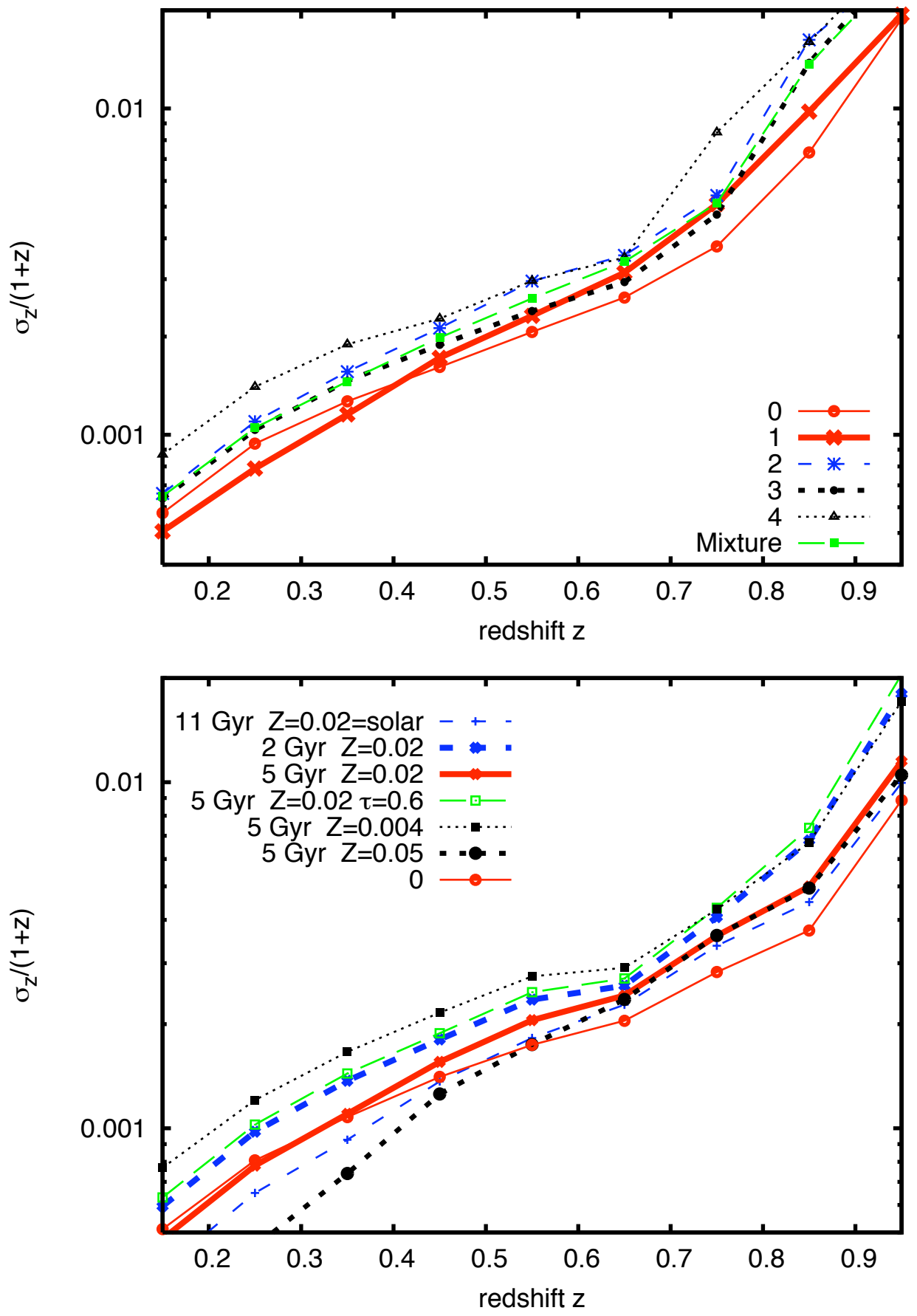

Figure 10. Top panel: Mock catalogs of $L_{*}$ galaxies are generated with one template for each line (0-4) and fitted with the five templates. We also show the case where the mock catalog is generated by random linear combinations of the five templates and fitted with the five templates. Bottom panel: Photo-z accuracy for spectra of single starbursts of fixed age and metallicity and no dust, compared with template 0 . The accuracy improves with increasing age and metallicity, and worsens with even trace amounts of star formation, but depends also on details of the modeling of giant and horizontal branch stars. 
the error when random linear combinations of the five templates are used to generate the galaxy photometry. As expected, the error obtained is an average of the errors from the templates 0 to 4 .

To understand the reasons why the errors depend on the template used, we plot in the bottom panel of Figure 10 the photo-z error for a set of templates computed for a stellar population of fixed age $(11,5$ and $2 \mathrm{Gyr})$ and metallicity $\left(Z_{\odot}, 0.2 Z_{\odot}\right.$, or $\left.2.5 Z_{\odot}\right)$; a template with an exponentially decaying star formation rate with $\tau=0.6$ Gyr at age $5 \mathrm{Gyr}$ is also included. These set of templates have been generated with the model of Bruzual \& Charlot (2003), and are used only in this figure for illustrative purpose. Smaller errors are generally obtained for stellar populations that are older and of higher metallicity. The presence of even a small amount of young stars (the model shown has a star formation rate that has decreased by a factor $e^{-5 / 0.6}=2.4 \times 10^{-4}$ since star formation started) increases the errors. There are, however, other factors affecting the photo-z errors in addition to the age and metallicity of the stellar population. In particular, the modeling of the giant and horizontal branch stars affects the part of the spectrum near the $\mathrm{H} \alpha$ break, and has a complex effect on the redshift errors. We note that, for $z>0.5$, template 0 has smaller errors than all other templates. The variations between templates 0 to 4 are due to differences in the distribution of stellar ages, amount of dust absorption, and different stellar evolution models used. Let us recall that templates 0 to 4 are built using the empirically derived Panter et al. (2007) star formation histories. Template 0 uses the SPEED models [21, which have been empirically found to provide a better fit to observations of LRG spectra than other stellar population models. Despite having the oldest stellar population, template 0 is bluer than template 1 in the rest-frame wavelength range of 4000 to $9000 \AA$ because it has a less developed giant branch and has no dust extinction. Template 1 aims at reproducing the spectrum of an S0/Sa galaxy, and has some dust absorption. Templates 1 to 4 are built using Charlot \& Bruzual 2008 models, which have been empirically shown to provide a better fit to Sa-Sc galaxies. Their larger errors compared to template 0 are due to different star formation histories (which vary among the templates in a complex way) and the different stellar evolution model. We note also that the redshift error depends on the band used to normalize the templates to the same luminosity; here we have normalized them to the same B-band luminosity, which is used to measure the luminosity function [19.

The variation of the errors with the assumed stellar population in each template is roughly associated with the decreasing amplitude of the $\mathrm{H} \alpha$ break for younger and less metal-rich galaxies, and with the addition of trace amounts of star formation (see $\S 5.1$, and eq. 77). For the same reason, changes in the modeling of giant and horizontal branch stars that affect the change of the $\mathrm{H} \alpha$ break have an important impact on redshift errors. Despite the complex dependence of the photo-z errors on several properties of the stellar population, the expected evolution with redshift is clear: stellar populations in LRG should be younger, star formation rates should increase, and the LRG population should become less homogeneous, leading to increased redshift errors. For similar reasons, redshift errors should decrease with galaxy luminosity. There is growing observational evidence that the most massive galaxies contain the oldest stellar populations up to $z \sim 1-2$ and they have less than $1 \%$ of their present stellar mass formed at $z<1$ [29, 30, 31, 22]. In high-density regions (i.e., galaxy clusters), massive systems ceased their star formation by $z \sim 3$ 31. While galaxies with stellar masses $>5 \times 10^{11} \mathrm{M}_{\odot}$ (roughly corresponding to $2 L_{*}$ ) ended their star-formation activity by $z \sim 2$, less massive objects were still actively 


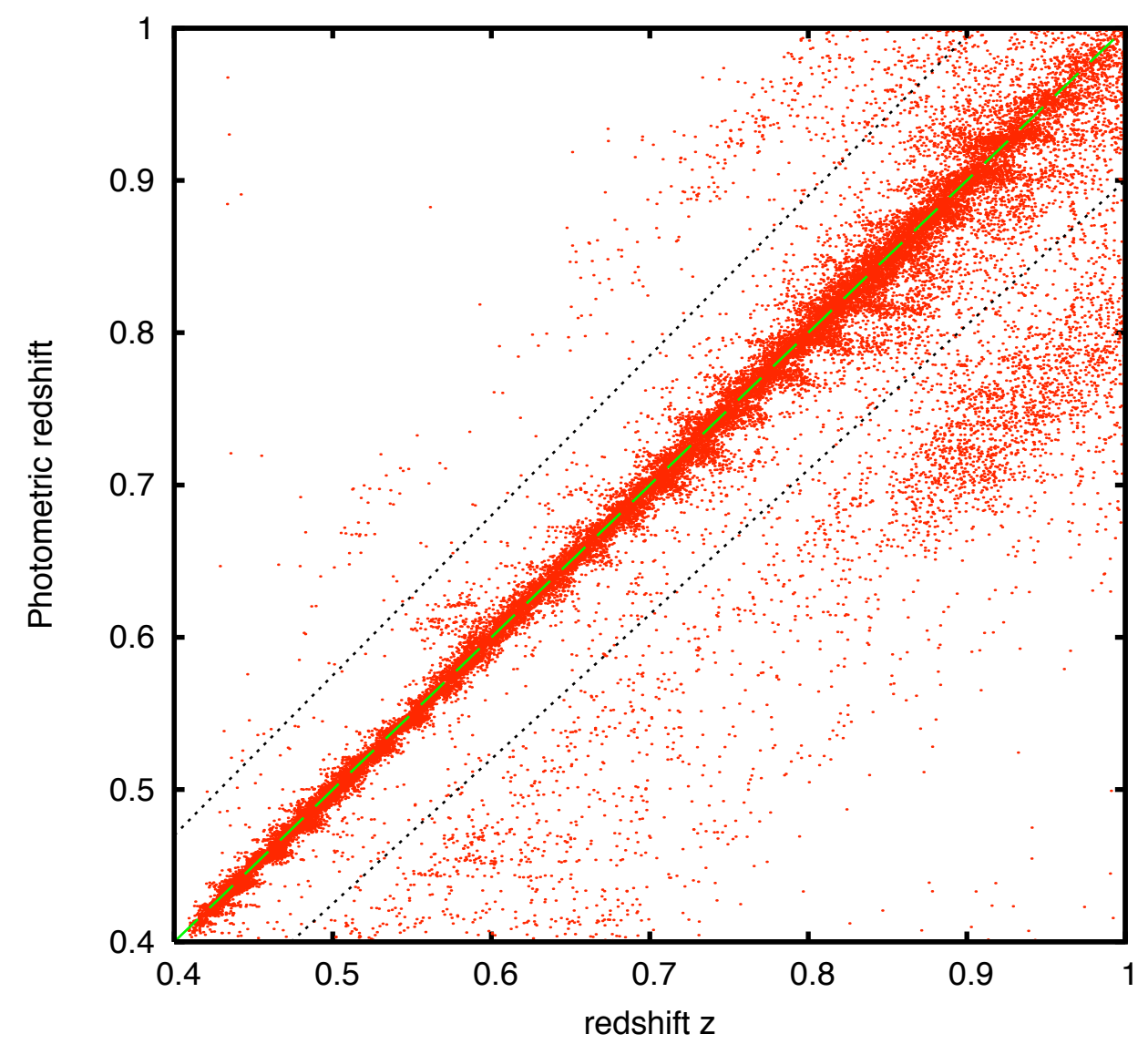

Figure 11. Photometric redshift versus input redshift. Our sample is generated by redshifting the spectra of SDSS DR6 LRG for $L=L_{*}$ and our fiducial survey (see text), and fitting them with our five templates. Dotted lines show the $\pm 0.05(1+z)$ photometric error range. All points outside the dotted lines are considered outliers that could eventually be removed.

forming stars at $z \sim 133$. As a result, the redshift errors found here using SDSS spectra of LRG, which are at $z \lesssim 0.4$ and $L \gtrsim 3 L_{*}$, are a lower limit to the redshift errors that may be found at higher redshifts and lower luminosities.

\subsection{Effects of variability in real LRG: SDSS Spectra}

In practical observations, the spectra of galaxies for which photometric redshifts are obtained are never exactly the same as the templates used for the fits. Real galaxy spectra are affected by the distribution in age, metallicity and dust obscuration, are not matched well enough by our imperfect models of stellar evolution, and may be substantially changed by very small amounts of star formation. For example, the presence of a weak OII emission line (at a wavelength close to that of the $\mathrm{H} \alpha$ break) due to traces of star formation may change the recovered photo-z. In addition, superposed light from very faint galaxies along the line-of-sight may introduce small changes in the spectra. Therefore, the redshift errors we found so far by assuming that the adequate 


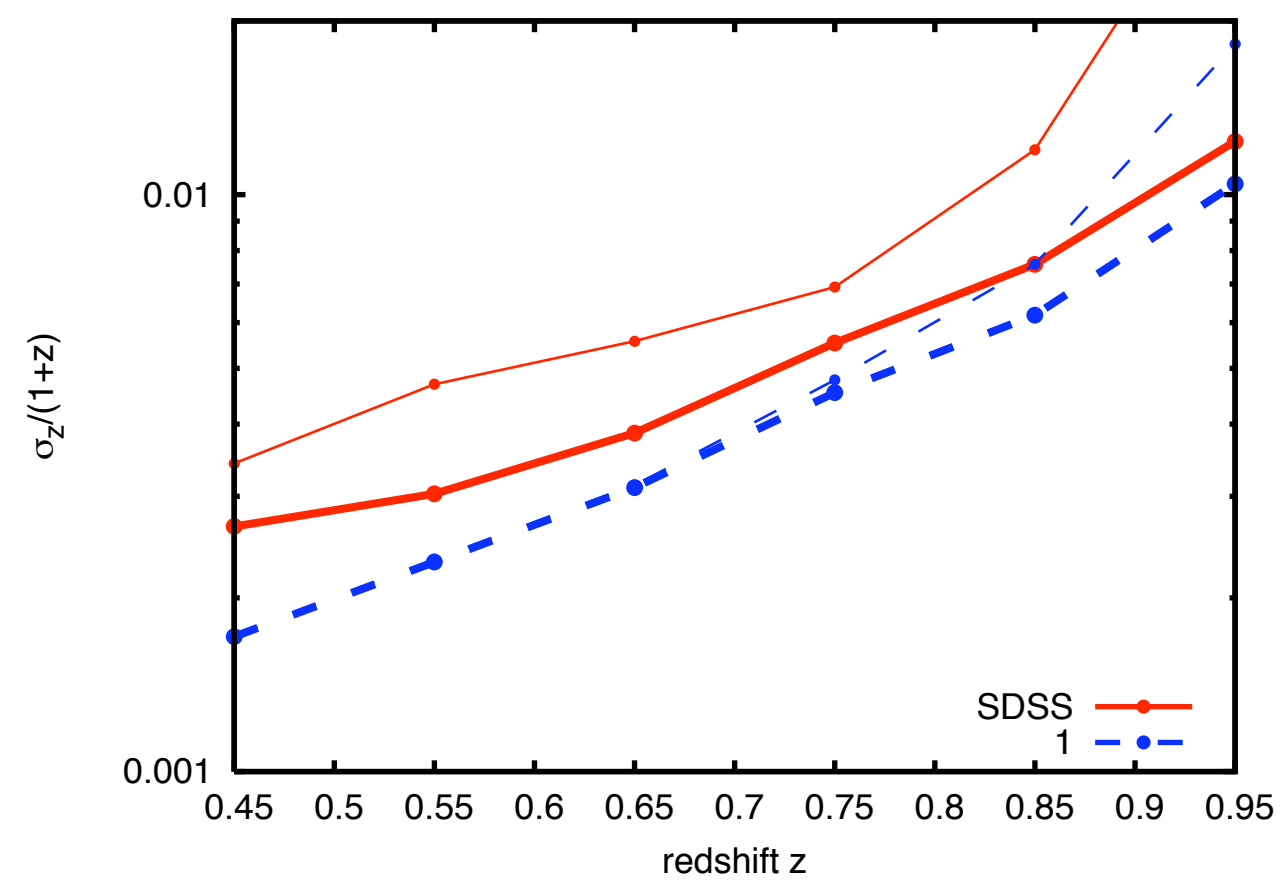

Figure 12. Solid lines: Photo-z error for redshifted SDSS LRG at $L=L_{*}$ in our fiducial survey, fitted with our five templates. The thin line shows the $68 \%$ error in each redshift interval of width $\Delta z=0.1$ without removing any outliers, and the thick line shows the same error when the outliers outside the range of the dotted lines in Figure 11 are removed. Dashed lines: Same results when we use a mock catalogue generated with template 1 . The recovered photo-z errors for observed SDSS increase relative to the case of ideal galaxies generated exactly with one of the five templates used.

templates are perfectly known may be severely underestimated.

The best and most extensive available survey of LRG is the SDSS. In this Section we use observed spectra of LRG obtained from the publicly available SDSS-DR6; spectro.princeton.edu catalog. We randomly select 50, 000 galaxies flagged as LRG for which a high signal-to-noise spectrum is available. Unfortunately, most of the SDSS LRG are at redshifts $z<0.4$. To simulate the measurement of a photometric survey at higher redshifts, we shift all the observed SDSS spectra by a constant factor of $f=530 / 380=1.395$ to longer wavelength. Hence, the true redshifts of the galaxies, $z$, are all shifted to a redshift for the simulated galaxy $z^{\prime}=(1+z) f-1$. With this shift factor, the minimum observed wavelength in the SDSS spectra, at $\lambda=380 \mathrm{~nm}$, is shifted just to the blue edge of our first filter at $530 \mathrm{~nm}$, and the redshift range shifts to the interval $0.4<z<1.0$, the most interesting range for measuring $\mathrm{BAO}$ with LRG photometric redshifts. We then follow the same procedure described in $\S 4$ : the shifted spectra are convolved with the filter window functions, noise is added to the fluxes at each filter, and the simulated photometry is fitted with our five templates.

We first examine a scatter plot of the fitted photometric redshift versus the true redshift of the SDSS galaxies (after the constant shifting mentioned above) for $L=L_{*}$ and our fiducial survey with $N_{f}=30$, in Figure 11. For guidance, a dashed line for 
$z=z_{\text {phot }}$ has been added. The two dotted lines indicate $1+z_{\text {phot }}=(1 \pm 0.05)(1+z)$, and will be our threshold for considering a photometric redshift as an outlier. The effect of the oscillations in the redshift error distribution with a period equal to the filter width, mentioned in $\S 5.2$, is also seen here. The dotted lines clearly separate a group of outliers in the error distribution, which arise because the $\mathrm{H} \alpha$ break is confused with other wider features in the spectrum when the noise is high. In the rest of this section, the results for the 68 percentile of the redshift error distribution will be shown for two different cases: considering the whole redshift error distribution, and removing the outliers defined as the points outside of the two dotted lines. The errors computed after removing the outliers should be considered as a highly optimistic case where the outliers are assumed to be perfectly identified with the help of additional broad-band filters in the survey.

The solid lines in Figure 12 are the 68 percentile redshift error as a function of redshift, for $L=L_{*}$ and our fiducial survey with $N_{f}=30$, as in Figure 11. The thin line is the value for the whole distribution, and the thick line is obtained after the outliers are removed. The effect of the outliers is substantial, increasing the redshift error by $\sim 40 \%$. For comparison, we show as dashed lines the case where galaxies generated with template 1 are fitted allowing for a linear combination of all our five templates, also for the cases of removing the outliers (thick line) or not removing them (thin line). Note that the thin dashed line is not exactly the same one as the thick solid line in Figure 9, the reason is that here, the 68 percentile error is found over each redshift interval of width $\Delta z=0.1$, in the same way as for the SDSS galaxies, whereas in previous plots the 68 percentile error is found first in bins of width $\Delta z=0.002$ and then averaged in quadrature over the larger width $\Delta z=0.1$. The errors are slightly reduced for this reason in Figure 12, and in reality one would have to deal in some way with the large oscillations with redshift shown in Figure 8, which are the main reason for the change in the error with the redshift bin width used for extracting the 68 percentile value. Even after the outliers are removed, the redshift errors obtained when fitting the realistic LRG population are substantially increased relative to fitting an ideal population matching template 1 exactly. An error below $0.003(1+z)$ is obtained only at $z<0.55$ for $L=L_{*}$. The rms error is still larger, because even after removing the outliers outside the dotted lines of Figure 11 the error distribution is still substantially non-Gaussian. Figure 12 also shows that the number of outliers is much smaller for galaxies generated with template 1. Real galaxy variability introduces complications in fitting photometric redshifts that increase the fraction of outliers.

Overall, the 68 percentile redshift error for $L_{*}$ galaxies increases by about $20 \%$ for SDSS galaxies compared to template 1 galaxies after the outliers are removed, and by $\sim 40 \%$ if the outliers are not removed. Comparing also to the results when template 1 galaxies are fitted with the same template 1 in Figure 9, we find that the redshift errors for the SDSS galaxies are $50 \%$ to $60 \%$ larger than the errors found when assuming that there is no galaxy variability at all, after removing the outliers, and a factor of 2 larger when outliers are not removed.

The redshift errors after removing outliers are also shown in Figure 13, as a function of galaxy luminosity, for several redshifts. Results are presented for the SDSS galaxies and for the cases of fitting template 1 galaxies with either template 1 alone or all our five templates. The error in the SDSS galaxies decreases much more slowly with luminosity than for mock galaxies generated with the templates. This is simply because the variability of SDSS LRG spectra is not well represented by our 


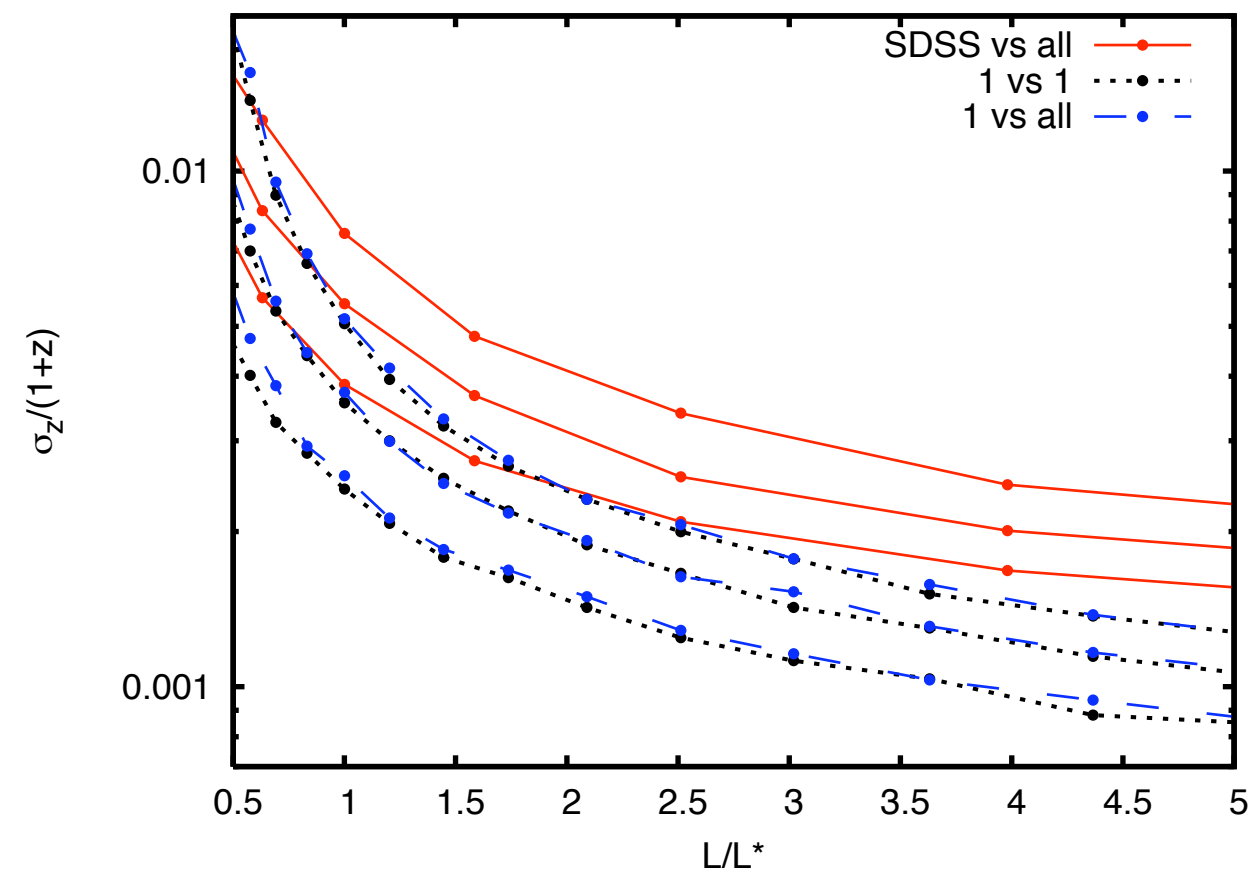

Figure 13. Photo-z error as a function of luminosity. Solid lines: Redshifted SDSS DR6 galaxies are fitted with our five templates. Dashed lines: Same results when galaxies are generated with template 1, and fitted with five templates. Dotted lines: Same results for galaxies generated with template 1 and fitted with the same template. The redshift error is the 68 percentile of the averaged distribution over each redshift interval of width $\Delta z=0.1$, centered at $z=0.65$, 0.75 and 0.85 (errors increase with redshift).

templates. For $L \gtrsim 2 L_{*}$, the errors for the SDSS galaxies are nearly twice as large as the errors obtained from galaxies that match template 1 exactly, while the errors when fitting with one or all five templates become nearly equal. The fraction of outliers is also found to be much larger for the SDSS galaxies. The galaxy variability in the SDSS LRG is highly complex and cannot be easily accounted for with a small number of templates, even when these templates have been calibrated with observed galaxies.

We have checked the dependence of the redshift errors on the signal-to-noise reached in the photometry. For $N_{f}=30$, we find that the photo-z error versus the signal-to-noise in the reddest filter $\left(S / N_{\text {last }}\right)$ is quite robust to the various assumptions made about the survey design. The target accuracy of $\sigma_{l z}=0.003$ is reached for SDSS galaxies at $S / N_{\text {last }} \simeq 20$, and at $S / N_{\text {last }} \simeq 12$ in the one template anlaysis. The photo$\mathrm{z}$ error and the fraction of outliers increase rapidly for lower signal-to-noise. At higher signal-to-noise, the errors continue to decrease rapidly for galaxies generated with the templates, but they flatten out to an "error-floor" of about $\sigma_{l z}=0.002$ for the SDSS galaxies, the "knee" being at around $S / N_{\text {last }} \sim 30$, indicating an intrinsic galaxy variability that is not adequately modelled by a small number of templates.

A possible problem when using the SDSS spectra of LRG to model photometry might arise from spectroscopic calibration errors, which could result in artificial variations of the simulated fluxes that are not due to real galaxy variability but 
to calibration errors. In fact, [16 argued that SDSS spectra cannot be used to model variability for this reason; we reach a different conclusion. Even though the spectroscopic calibration errors are found to be typically at the level of $\sim 4 \%$ on wavelength scales of $100 \mathrm{~nm}$ when comparing photometry and spectroscopy of stars (see http://www.sdss.org/dr6/products/spectra/spectrophotometry.html ), the precision of the photometric redshifts depends on measuring spectral features at the smallest wavelength scale allowed by the narrow-band filters (for LRG, this is mostly the $\mathrm{H} \alpha$ break). For a filter width of $10 \mathrm{~nm}$, the derived photometric redshifts might be affected by spectroscopic calibration errors on a similar wavelength scale. At this scale, the calibration errors are much smaller: they are certainly less than $2 \%$ from the analysis of quasar spectra in regions with no Ly $\alpha$ forest absorption (P. McDonald, private communication; see also Figure 19 in [32, where the contribution to the flux variance due to calibration errors from different scales in quasar spectra is shown as a ratio to the $\operatorname{Ly} \alpha$ forest power), and they should be less than $1 \%$ from the most recent calibration made with low-metallicity F-subdwarfs (D. Schlegel, private communication). We therefore conclude that the variations in the SDSS spectra of LRG galaxies which result in increased photometric redshift errors are mostly real and not due to spectroscopic calibration errors.

We emphasize that, owing to galaxy evolution, the variability present in the SDSS spectra is a lower limit for the variability that should be expected for LRG at higher redshift, as discussed in $\S 5.4$. The errors might possibly be reduced by further optimization of the templates as a function of galaxy luminosity and environment if galaxy variability at high redshift were better understood, but how substantial an improvement may be achievable in this way remains to be proven.

\section{Discussion}

This paper has presented an analysis of the expected photometric redshift errors in LRG that can be achieved in an imaging survey with a system of a large number of narrow-band filters. We have found that an optimal choice for the number of filters is $N_{f} \simeq 30$ over the wavelength range 530 to $830 \mathrm{~nm}$, allowing redshift measurements over the range $0.5<z<0.9$. A central focus of our investigation is that the intrinsic variability of LRG implies a substantial increase of the redshift errors compared to a calculation where all the LRG are assumed to match exactly the same template used to fit their photometry. We find that it is crucial to properly include the effects of spectral variability when evaluating the prospects for any survey to measure BAO (particularly in the radial direction) in the power spectrum of LRG.

The implications of our results can be summarized by considering, for any particular survey, the lowest luminosity that a galaxy needs to have to yield a redshift accuracy better than the target level for measuring BAO in the radial direction, $\sigma_{l z}<0.003$. The power of a survey to reach the required photometric accuracy depends mainly on the product $E t$ of the etendue times the total observing time. This lowest luminosity as a function of $E t$ is shown in the upper panel of Figure 14, at five different redshift intervals, when the total survey area is fixed to $\Omega=8000$ square degrees and $N_{f}=30$, for the SDSS galaxies after removing outliers with redshift errors $\Delta z>0.05(1+z)$. Note that the minimum luminosity shown at each value of $E t$ is the one that would yield a 68 percentile error equal to $\sigma_{l z}=0.003$; the rms error would be larger because even after removing the most extreme outliers, the error distribution is still substantially non-Gaussian. The luminosity function [19] implies the number 

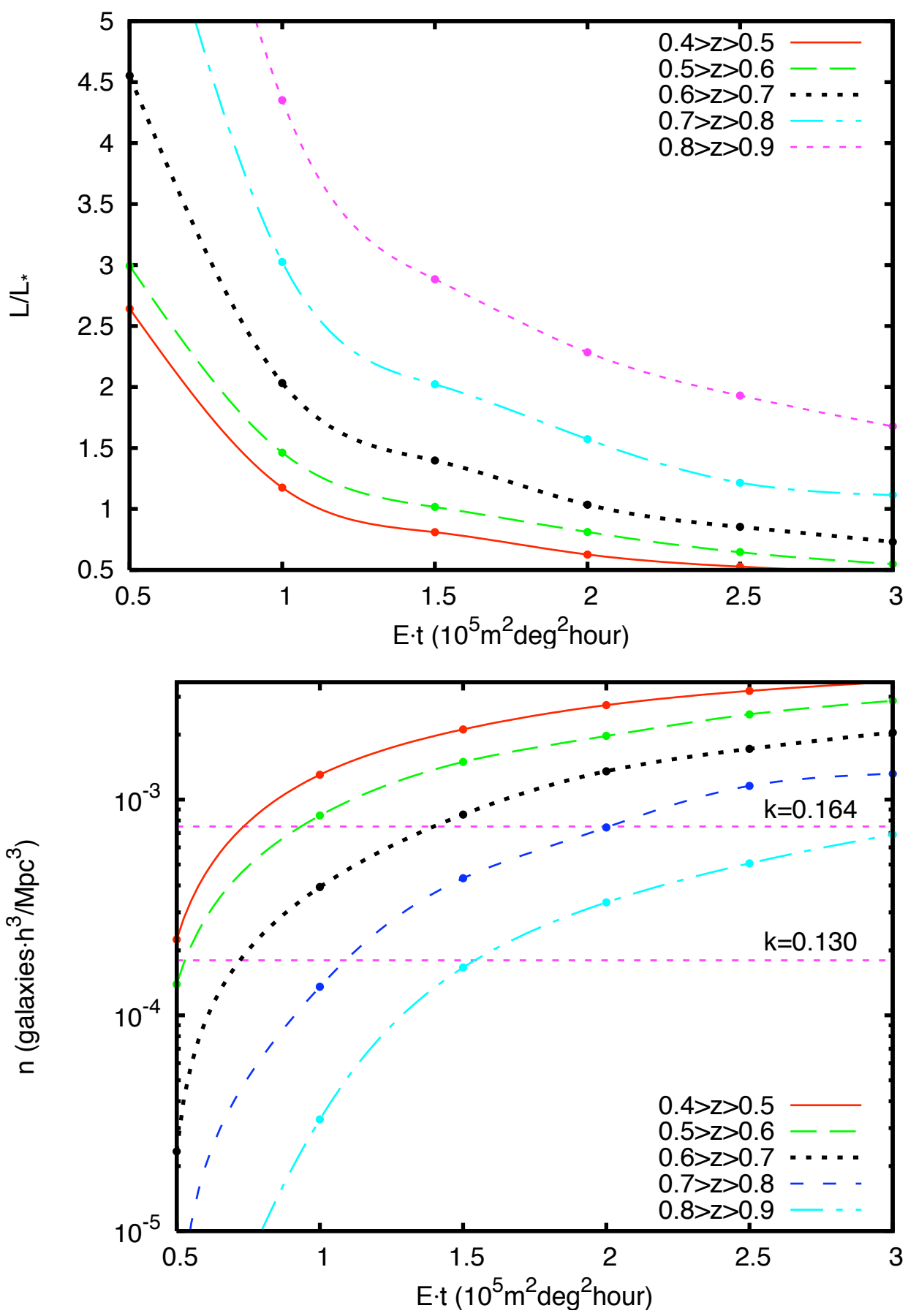

Figure 14. Top panel: Luminosity of an LRG with photometric redshift error $\sigma_{l z}=0.003$ after removing outliers, as a function of the etendue $\times$ observing time of the survey, for the five redshift intervals indicated. Errors are computed using the spectra of SDSS LRG. Bottom panel: Corresponding number density of galaxies with a photometric redshift error better than $0.003(1+z)$, as a function of $E t$, from the luminosity function [19] after taking out the fraction of outliers. Horizontal dashed lines indicate the required galaxy densities for reaching a shot noise with $n P=1$ at two wavenumbers (differing by a factor of two in the total number of modes they comprise), where $P$ is the line-of-sight power spectrum assuming $\sigma_{l z}=0.003(1+z)$ and shown in Figure 1 Our fiducial survey has $E t=1.5 \times 10^{5} \mathrm{~m}^{2} \operatorname{deg}^{2} \mathrm{hr}$. 
density of galaxies above this minimum luminosity that is shown in the bottom panel, after subtracting outliers. As shown in Figure 1, the photometric redshift errors suppress the power spectrum near the line-of-sight, which must be compensated by an increased number density of galaxies to keep shot noise sub-dominant on a given scale. The bottom panel of Figure 14 addresses this issue. For redshift errors $\sigma_{l z}=0.003$, the two horizontal dashed lines show the galaxy number density required to reduce the shot noise to the level $n P=1$ at wavenumbers $k=0.13 h \mathrm{Mpc}^{-1}$ and $k=0.164$ $h \mathrm{Mpc}^{-1}$ near the line-of-sight. Modes up to $k=0.164 h \mathrm{Mpc}^{-1}$ must be sampled to fully measure the second BAO. Figure 14 shows that Et $>3 \times 10^{5} \mathrm{~m}^{2} \operatorname{deg}^{2} \mathrm{hr}$ is required to reach $n P=1$ up to $z=0.85$ for $k=0.164 h \mathrm{Mpc}^{-1}$ near the line-ofsight. For the characteristic total observing time of 5000 hours assumed in our fiducial survey, this corresponds to $E>60 \mathrm{~m}^{2} \mathrm{deg}^{2}$.

This minimum value for $E t$ depends on several technical details of the telescopecamera system and the observing conditions for the survey. The assumptions made here for our fiducial survey were specified in $\S 4$ : a seeing of 0.8 ", read-out noise of $7 \times 3$ electrons per pixel (with a pixel size of 0.4 "), the efficiency for the latest available CCD's, optical losses of two mirror reflections only, and the sky brightness of the Paranal observatory at an airmass of 1.2. These assumptions can be considered as highly optimistic, except perhaps the assumption on the read-out noise which might be improved with the best available technology. Changes in these assumptions imply corresponding changes in the required value of Et necessary to reach a fixed signalto-noise in the galaxy photometry. We can quantify this in the following way:

- A seeing degradation from 0.8 " to 1.2 " implies a $20 \%$ decrease in the signalto-noise for a fixed aperture, and therefore requires a $40 \%$ increase in Et $\left(d \ln E t / d \ln\right.$ seeing $\left.g^{\prime \prime} \simeq 1\right)$

- An increase by half a magnitude of the sky brightness (comparable for example to the sky variation over a sun cycle or between ecliptic plane and ecliptic pole) can be compensated by a increase in $E t$ of a factor $1.6\left(d \ln E t / d B_{s k y} \simeq 0.9\right)$

- A reduction in readout noise of a factor of three can be compensated by a $\sim 20 \%$ reduction in $E t$ ( $d \ln E t / d \ln N_{\text {readout }}=0.3$; we have verified this by repeating all the above calculations for a lower read-out noise). An even lower read-out noise, however, would not yield the same fractional improvement because at that point the read-out noise becomes subdominant.

- Any factors that decrease the overall throughput of the system (i.e., lower efficiency of the CCD's, additional optical losses in the telescope-camera system, reduced filter transmission, etc.) will of course increase the required $E t$ in proportion to the inverse of the throughput. An important factor that may cause such a reduction in the throughput would likely be present in a system of narrow filters based on interferometry, where the filter window has a variation across the field-of-view caused by the varying incidence angle of the light. The averaging of the filter window shape as a source moves across the field in drift-scanning mode would widen the filter window without increasing the number of detected photons.

These considerations mean that, in practice, the required etendue would likely be substantially larger. 


\section{Conclusions}

The principal objective of this paper is to examine the potential for narrow-band photometric surveys to measure the radial BAO signal in the LRG power spectrum. We have revisited this issue after [12, 11, 14, addressing the optimization of the number of filters and filter width, discussing the dependence on the spectra of the target population of LRG, and considering the signal-to-noise that could be realistically achieved in a survey. We have found it to be particularly important to take into account the spectral variability of a realistic galaxy population for properly evaluating the photometric redshift accuracy that can be achieved. Our conclusions can be summarized as follows:

a) In agreement with 12, 11, 14 the photometric approach, even if it can achieve the target photo-z error of $0.3 \%$, will be advantegeous only if it can cover a much larger fraction of the sky, with a higher galaxy density and reach higher redshifts than the spectroscopic surveys currently under way. Not only one needs higher source density in a photometric survey to measure the oscillations up to $k \sim 0.2$, but also the distribution of redshift errors needs to be known accurately to correct for their effect on the power spectrum shape and be able to measure the BAO shape. This is equivalent to the requirement of obtaining photo-z with better than $0.3 \%$ accuracy to fainter magnitudes than spectroscopy. We have concentrated on the LRG galaxies because they are a fairly homogeneous population, the form of their spectra makes them particularly suitable for good photo-z determinations and because they represent the target of choice for $z<2$ BAO surveys.

b) If LRG were a perfectly homogeneous population (with spectra closely matched by a single spectral template), the photometric redshift accuracy would not improve very much beyond a total number of filters $N_{f} \simeq 10$, except for galaxies at high signal-to-noise which would have a low number density in a survey with $E t \simeq$ $1.5 \times 10^{5} \mathrm{~m}^{2} \mathrm{deg}^{2} \mathrm{hr}$. In reality, galaxy spectra are variable and the ideal number of filters is $N_{f} \simeq 30$ over a wavelength range $530 \mathrm{~nm}<\lambda<830 \mathrm{~nm}$, focusing on the redshift range $0.5<z<0.9$. Medium-to-narrow band filters photometric redshift errors show periodic oscillations with a spacing $\delta z$ that corresponds to the filter widths. For a filter width of $\sim 100 \AA$, the scale of the oscillation corresponds to the BAO scale. The presence of these oscillations may introduce significant biases in the measurement of the BAO scale (see Section 5.2).

c) The variability of realistic galaxy spectra demand high resolution (or more filters in a photometric survey) and high signal-to-noise. We have quantified, with realistic simulations of a photometric survey, the minimum galaxy luminosity and the corresponding number density for which $0.3 \%$ photo-z error can be obtained as a function of the survey Etenduex exposure time Et (see Figure 14). We estimate the minimum value of $E t$ necessary to measure the full second baryonic acoustic oscillation in the power spectrum, at $k<0.164 h / \mathrm{Mpc}$ near the line-of-sight, with shot noise $n P>1$ and up to $z=0.85$, at $E t \simeq 3 \times 10^{5} \mathrm{~m}^{2} \mathrm{deg}^{2} \mathrm{hr}$. The actual required value of $E t$ would likely be larger when taking into account that our assumptions for the seeing and the overall throughput of the telescope-camera system are optimistic, and that the presence of outliers, optimistically clipped and ignored, will degrade the estimated performance. Moreover, the true level of galaxy variability should likely be higher than in the SDSS LRG sample we have used (which is for $z \lesssim 0.4$ and $L \gtrsim 3 L_{*}$ ), because the variability of LRG spectra is expected to increase with redshift and decrease with luminosity. 
Current spectroscopic surveys (e.g., SDSSIII-BOSS) are obtaining spectra of the massive $\left(>2 L^{*}\right)$ population of LRG at $z<0.75$ to measure radial and tangential $\mathrm{BAO}$ scale. Here we have investigated the conditions necessary to obtain the required photo-z accuracy of $0.3 \%$ from a similar or fainter population using photometry, which in principle is less expensive in terms of observing. We have concluded that an etendue of $30 \mathrm{~m}^{2} \mathrm{deg}^{2}$ is required for tracing the same population as BOSS $\left(z<0.75, L>2 L^{*}\right)$ with radial BAO signal degraded only by $\sim 60 \%$ due to photo-z errors. But to improve on this i.e., to reach $L_{*}$ galaxies at slightly higher redshifts, the minimum etendue required is $60 \mathrm{~m}^{2} \mathrm{deg}^{2}$ for a 5-year survey (corresponding to a $4 \mathrm{~m}$ telescope with the largest fields of view that have been made), and probably needs to be larger given our optimistic assumptions we have made for the seeing, throughput, observing time, removal of outliers and systematic errors.

In this paper we have restricted our attention to the objective of measuring radial BAO with LRG. However, a large-area imaging optical survey with a large number of narrower bands than existing surveys would have many other applications, and might lead to a large number of interesting astronomical discoveries. We have shown in this paper that, for realistically achievable values of $E t$ at the present time, the density of LRG sources that could be measured with a narrow-band photometric survey with the target redshift accuracy for radial $\mathrm{BAO}$ will not be large enough to make it competitive with a spectroscopic survey. Therefore, a photometric survey with a large number of optical narrow-bands needs to find its scientific justification in other astronomical applications.

\section{Acknowledgements}

We warmly thank D. Eisenstein, J. Gunn, P. McDonald and D. Schlegel for discussions. This work was carried out in the framework of the PAU Consolider Collaboration: the authors are part of the Physics of the Accelerating Universe (PAU) proposal, currently supported by the Spanish Ministry for Science and Innovation (MICINN) through the Consolider Ingenio-2010 program project CSD2007-00060. DR acknowledges support from the Spanish MICINN through a FPU grant. LV acknowledges the support of FP7-PEOPLE-2002IRG4-4-IRG\#202182 and CSIC I3 \#200750I034. The work of RJ is supported by grants from the Spanish MICINN and the European Union (FP7). JM is supported by the Spanish MICINN grants AYA2006-06341 and AYA-15623-C02-01 and the European Union FP6 grant IRG-046435. CPG acknowledges MICINN grant No. FPA2007-60323.

\section{References}

[1] Simon J., et al., 2005, PhRvD, 71, 123001

[2] Komatsu E., et al., 2008, arXiv:0803.0547

[3] Polarski D., Ranquet A., 2005, PhysLettB, 627, 1

[4] Huang Z.Y., Wang B., Su R. K., 2007, Int J.Mod.Phys. A 22, 1819

[5] Clarckson C., Cortes M., Bassett B., 2008, JCAP 0780011

[6] Baremboin G., Fernandez-Martinez E., Mena O., Verde L., in preparation.

[7] Eisenstein D. J., et al., 2005, ApJ, 633, 560

[8] Cole S., et al., 2005, MNRAS, 362, 505

[9] Percival W., et al. 2007a, MNRAS, 381, 1053

[10] Percival, W., et al. 2007b, ApJ, 657, 645

[11] Seo, H.-J., \& Eisenstein, D. J. 2007, ApJ, 665, 14

[12] Seo H.-J., Eisenstein D. J., 2003, ApJ, 598, 720 
[13] Wolf C., Meisenheimer K., Rix H.-W., Borch A., Dye S., Kleinheinrich M., 2003, A\&A, 401, 73

[14] Blake C., Bridle S., 2005, MNRAS, 363, 1329

[15] Dahlen, T., et al., 2008, AJ, 136, 1361

[16] Benitez N., et al., 2008, arXiv:0807.0535

[17] McDonald, P., \& Eisenstein, D. J. 2007, PRD, 76, 063009

[18] Kaiser, N. 1987, MNRAS, 227, 1

[19] Brown M. J. I., et al., 2007, ApJ, 654, 858

[20] Niemack M. D., et al.., 2008, arXiv:0803.3221

[21] Jimenez R., et al., 2004, MNRAS, 349, 240

[22] Panter, B., et al., 2007, MNRAS, 378, 1550

[23] Shen, S., et al., 2003, MNRAS, 343, 978

[24] Bruzual G., Charlot S., 2003, MNRAS, 344, 1000

[25] Patat, F. 2008, A\&A, 481, 575

[26] Roy A. E., Clarke D., 2003, Astronomy, IoP Publishing

[27] BARR Associates, 2008, private communication

[28] Fabricius M. H., et al., 2006, SPIE6068.

[29] Cowie, L. L., Songaila, A., \& Barger, A. J. 1999, AJ, 118, 603

[30] Heavens A., et al., 2004, Nature, 428, 625

[31] Thomas D., et al., 2005, ApJ, 621, 673

[32] McDonald, P., et al. 2006, ApJS, 163, 80

[33] Treu T. et al., 2005, ApJ, 633, 174 